\title{
Translational specialization in pluripotency by RBPMS poises
}

\section{future lineage-decisions}

Deniz Bartsch ${ }^{1,4,5}$, Kaustubh Kalamkar ${ }^{1,4,5}$, Gaurav Ahuja ${ }^{1,3,4,5}$, Hisham Bazzi ${ }^{5}$, Argyris Papantonis ${ }^{2}$ and Leo Kurian ${ }^{1,4,5, \#}$

${ }^{1}$ Center for Molecular Medicine Cologne (CMMC), University of Cologne, 50931 Cologne, Germany

2 Institute of Pathology, University Medical Center Göttingen, 37075 Göttingen, Germany

${ }^{3}$ Present address: Department of Computational Biology, IIIT Delhi, New Delhi 110020, India

${ }^{4}$ Institute for Neurophysiology, Faculty of Medicine, University of Cologne, 50931 Cologne, Germany

${ }^{5}$ Cologne Cluster of Excellence in Cellular Stress Responses in Ageing-associated Diseases (CECAD), University of Cologne, 50931 Cologne, Germany

\# Correspondence to: leo.kurian@uni-koeln.de

During embryonic development, translation is proposed to be uniquely specialized at the exit of pluripotency to rapidly reprogram the proteome and enable lineage commitment ${ }^{1-5}$. Yet, the fundamental role of translational specialization in programming developmental cell-fate decisions, its mediators and their mode-of-action remain elusive ${ }^{2,3}$. Here, using human embryonic stem cell-based models, we report that mesoderm commitment from pluripotency is controlled by the translational specialization factor RBPMS. RBPMS-driven translational specialization balances the abundance of cellfate regulators to authorize accurate lineage decisions upon receiving differentiation signals. Consequently, RBPMS loss, without affecting pluripotency, selectively and severely impedes mesoderm specification and subsequent cardiogenesis. Mechanistically, direct binding of RBPMS to 3'UTRs allows selective translation of developmental cell-fate regulators including integral morphogen signaling components specifying mesoderm. RBPMS loss disrupts the canonical composition of translation initiation complexes resulting in the aberrant retention of initiation factors on ribosomal complexes. Our data unveil how emerging lineage choices from pluripotency are controlled by translational specialization via ribosomal platforms acting as a regulatory nexus for developmental cell fate decisions. 
Embryonic development relies on precise and coordinated cell-fate decisions, a complex process that sculpts an entire organism from a single totipotent cell ${ }^{6-10}$. A central question in developmental biology is how genetic information is differentially interpreted to generate the multitude of cell-fates that constitute an organism. Cell fate transitions and the conferring of cellular identity are dictated by spatiotemporal control of the communication of genetic information ${ }^{6,8,11}$. In this regard, morphogen-mediated signal transduction, epigenetic, and transcriptional mechanisms have been the focus of research over the last decades ${ }^{12-14}$. However, in mammals, translational control is the primary determinant of protein abundance ${ }^{1,15,16}$. Therefore, success of developmental cell fate decisions relies on the efficient rewiring of the proteome to support rapid cellular identity changes, indicative of a vital role for regulation at the translation level ${ }^{2,3}$. Translational control is achieved by rapid and dynamic regulation of global translation rates and by selective translation of specific mRNA subsets, termed "translational specialization"17-20. Translational specialization is highly context-specific, and proposed to be vital for regulating cell fate decisions during development ${ }^{21-25}$. Studies using mouse ES cells (mESCs) revealed an immediate and substantial increase in protein synthesis upon induction of differentiation; this indicates a poorly understood, systems-wide, reprogramming of the translatome ${ }^{4}$. Elegant in vivo studies suggested that at the exit of pluripotency, mesoderm lineage is particularly dependent on translational control ${ }^{26}$. Translational control was also shown to be of paramount importance during maternal-zygotic transition and in the regulation of Hox mRNAs $22,27-29$. These reports, along with mass spectrometry-based studies analyzing ribosome composition in $\mathrm{mESC}$, indicated the importance of translational control during early embryonic development ${ }^{30}$. Translational specialization is thought to be conferred by translation specialization factors (TSFs) that dynamically sequester on ribosomal complexes to program the selective and privileged translation of their target $m R_{N A s}^{3,31,32}$. However, to date, there is no systems-wide understanding of such regulators, of the molecular mechanism(s) or of the principles by which the developmental transcriptome is differentially translated in time and space to allow cell fate specification, especially in humans ${ }^{32}$.

\section{Unprecedented diversity of proteins residing on ribosomal complexes in hESCs}

We hypothesized that the translatome at the state of pluripotency is poised for rapidly responding to differentiation stimuli to reprogram the proteome to orchestrate lineage commitment ${ }^{5}$. Then, such translational reprogramming could be achieved by a crosstalk between TSFs that sequester on ribosomal complexes and as-yet-unknown RNA elements built into the transcripts of developmental regulators, together controlling ribosomal output. To identify TSFs that control cell fate decisions from pluripotency, we first systematically characterized the proteome of ribosomal complexes in human embryonic stem 
cells (hESCs). To achieve this, we isolated ribosomal fractions from hESCs corresponding to 40S, $80 \mathrm{~S}$ (monosome), and polysomes (light polysome fraction and heavy polysome fractions) under steady state conditions by polysome fractionation followed by liquid chromatography coupled mass spectrometry (LCMS) to identify their protein composition (translation state mass spectrometry, TS-MS) (Fig. 1a, detailed protocol in Methods). We filtered out all ribosomal proteins and known translation factors and identified 1380 proteins sequestering on ribosomal complexes at steady-state. Of these, 450 proteins were significantly enriched (fold change $\geq 2, p$-value $\leq 0.05$ ) on at least one of the ribosomal complexes, with a strong bias for the $40 \mathrm{~S}$ fraction, which harbors approximately half of all differentially-enriched proteins (Fig. 1b, Source Data Fig. 1). RNA binding proteins (RBPs) were the largest class of proteins (30-50\% of the proteins differentially associating with ribosomal complexes) in each ribosomal complex (Fig. 1b, Source Data Fig. 1). Next, we performed pathway enrichment analysis to evaluate the representative functional features of the candidate TSFs and grouped them based on their common functional features (Fig. 1c, Source Data Fig. 1). Proteins in each fraction exhibited discrete functional signatures, and overlapped with signatures in adjacent fractions, suggesting functional specialization and cooperativity. Candidate TSFs in the steady state included regulators of protein and nucleic acid metabolism, ribosome biogenesis, translation, cellular metabolism including electron transport chain, translation and translation regulation as well as RNA transport, suggesting a potential crosstalk amongst these fundamental processes on ribosomes.

Notably, our data indicate that ribosomal complexes are frequented by numerous functionally diverse protein groups, in line with previous reports from murine models ${ }^{30}$. This makes identification of functional TSFs highly challenging. Therefore, we reasoned that functional TSFs could differentially enrich on ribosomal complexes upon challenging translation to enable a selective, specialized translational state. As challenging translation by transient stress is known to rapidly bring the translatome into "survival mode" (where the majority of translation is significantly downregulated while proteins aiding survival and maintenance are selectively translated ${ }^{33-36}$ ), we exploited this using a 2-h treatment with $0.05 \mathrm{M} \mathrm{NaAsO}_{2}$. Of note, hESCs recover back to steady state from such a challenge within 4 hours. Evaluation of polysome profile traces of hESCs grown under steady state in comparison to translational stress showed a pronounced decrease of the $80 \mathrm{~S}$ and polysome peaks, denoting general translational inhibition (Extended Data Fig. 1a, b). Therefore, we reasoned that translation stress-induced enrichment of a given protein on ribosomal fractions could be used as a prioritization feature for putative, currently unknown, regulators of translation. We thus also performed TS-MS after challenging hESCs with $\mathrm{NaAsO}_{2}$ and analyzed the 
differential enrichment of robustly-identified proteins between steady state and translation challenge at each ribosomal fraction.

The reproducibility of such an approach is reflected by the low variance of the entire proteome in each biological replicate of ribosomal fraction upon principal component analysis (Extended data Fig. 1c). Importantly while all independent ribosomal complexes clustered together, the corresponding complexes upon stress showed marked separation to that of steady state level. These findings indicate that a transient translational challenge remodels the ribosome-associated proteome (Source Data Fig. 1). Nevertheless, the occurrence of ribosomal proteins in expected fractions across all samples further indicated that the isolated fractions indeed represent the indicated ribosomal complexes (Extended Data Fig. 1d). As we predicted, candidate TSF signatures were notably different upon translational challenge. Mainly the proteostasis machinery (on the 40S) and the RNA degradation machinery (on polysomes) were enriched, while proteins involved in metabolism were depleted (from 805 and polysomes) (Extended Data Fig. 1e, f). Importantly, substantially more RBPs sequestered on ribosomal complexes (most on polysomes) upon translational challenge (Extended Data Fig. 1f). Thus, ribosomal complexes in hESCs are bound by an array of candidate TSFs involved in a host of primary cellular processes, including energy metabolism, protein homeostasis, and RNA metabolism, substantial fraction of which are uncharacterized RBPs

\section{RBPMS is a candidate TSF in hESCs}

We focused on RBPs enriched on ribosomal complexes for investigating their role as TSFs considering their ability to directly interact with ribosomal complexes, as well as a broad network of mRNAs. In total, we identified 600 RBPs (excluding ribosomal proteins and known translation factors) as significantly enriched on translational complexes when all conditions were (Fig. 1d, Source Data Fig. 1). Interestingly, these constitute $\sim 24 \%$ of all known RBPs and $\sim 38 \%$ of the RBPs reported in the proteome of stress granules ${ }^{34,37-}$ 39. In line with our initial aim to identify factors that could play a central role in regulating translation, we reasoned that a functional TSF would show preferential, dynamic association with ribosomal complexes upon transient translational stress. We statistically evaluated the dynamic enrichment of these 600 RBPs with each of the ribosomal complexes in steady state and upon translational challenge and ranked them based on preferential enrichment upon stress (Hoteling's Two-Sample T2) (Fig. 1e, Source Data Fig. 1). The top-ranking candidate TSF, RNA binding protein RBPMS (RNA-Binding Protein with Multiple Splicing) was selected for further analysis. Notably, the top five enriched RBPs were BZW1 ${ }^{40}, A A R S^{41}, \mathrm{HMGB2}^{42}$, RBPMS, and KNOP1, of which all except RBPMS and KNOP1 (KNOP1 has been reported to be in the 
nucleolus ${ }^{43}$ ) are known regulators of translation, consolidating that our prioritization criteria do identify translational regulators. RBPMS is an evolutionarily conserved, but poorly characterized RBP suggested as a regulator of embryogenesis ${ }^{44-46}$. It is predominantly a cytosolic protein in hESCs (Fig. 1h), enriched in $40 S$ complexes in steady state, and moving into polysomal fractions upon translational challenge, suggesting a role in translational regulation (Fig. 1f, $\mathbf{1 g}$ ).

Because our method relies on density gradient separation of protein complexes followed by mass spectrometry, the protein complexes with similar sedimentation profiles as those of the translational machinery could be present in our dataset. Previous reports using mESCs reported membrane proteins, centrosomes, clathrin complexes, as well as the vault complex to be present in TS-MS data ${ }^{30}$. However, components of these complexes were scarce in our data and duly filtered out, although we cannot completely rule out transient interactions or shuttling of factors between these complexes and the translational platforms, which might be relevant (Extended Data Fig. 1g, Source Data Fig 1).

Thus, to confirm a direct association of RBPMS with ribosomal complexes, we used an orthogonal approach. We reasoned that if RBPMS associates with translational machinery in hESCs, then upon treatment with specific translation inhibitors, it would show a characteristic shift in its sedimentation profile corresponding to the step of translation that is inhibited. First, we used the specific translation initiation inhibitor harringtonine ${ }^{5}$. Following $2 \mu \mathrm{g} / \mathrm{ml}$ harringtonine treatment for $30 \mathrm{~min}$, RBPMS was depleted from polysomes and concomitantly enriched in initiation fractions; changes in the enrichment of bona fide components of the translation machinery, EIF4G, PABP, and RPL13 serve as a control (Fig. 1i, top two panels). We next treated hESCs with the inhibitor of elongation, puromycin $(1 \mu \mathrm{g} / \mathrm{ml}$ for $1 \mathrm{~h})$, to induce translational arrest. This led to a depletion of RBPMS across fractions (Fig. 1i, third panel). Next, RNase I (5U, $30 \mathrm{~min}$ ) treatment which disrupts ribosomal complexes, resulted in the accumulation of RBPMS in the $40 \mathrm{~S}$ fraction (Fig. 1i, last panels). To our knowledge, there are no other complexes that would show a similar sedimentation profile as ribosomal complexes and would simultaneously show the characteristic changes in the profile upon treatment with specific translation inhibitors. Considering its enrichment on the $40 \mathrm{~S}$ complex in steady state and upon various treatments with translation inhibitors, notably upon RNase I, we can rule out contamination with nascent RBPMS as highly unlikely. Taken together, also using an orthogonal approach, we identify RBPMS as a candidate TSF in hESCs.

\section{RBPMS loss impedes translation in hESCs}

To investigate the molecular and functional role of RBPMS, we targeted the exon-intron boundary of exon 1, using two gRNAs employing CRISPR/Cas9-mediated genome engineering to generate a complete 
knockout in hESCs (hereafter RBPMS-KO) (Fig. 2a, Extended Data Fig. 2a). The homozygous deletion of the exon-intron boundary disrupted the natural open reading frame of RBPMS resulting in a complete loss of function, confirmed both at the RNA and protein levels in comparison to the isogenic wild type hESCs (WT) (Fig. 2b, Extended Data Fig. 2b).

RBPMS-KO hESCS display significant polysome depletion, indicative of severe translational inhibition (Fig. 2c). This leads to $~ 50 \%$ reduction in overall protein synthesis in hESCs, indicated by the strong reduction of newly synthesized proteins detected by short-term (10 $\mathrm{min}$ ) puromycin labeling evaluated either using an anti-puromycin antibody or fluorescent azide conjugated O-propargylpuromycin, to avoid any detection dependent biases (Fig. 2d, e, Extended Data Fig. 2h).

Nevertheless, translation inhibition due to the loss of RBPMS did not affect hESC self-renewal or cell cycle (Extended Data Fig. 2c), their overall mitochondrial integrity or metabolism (Extended Data Fig. 2d-f), while global transcriptional output was marginally affected (EU incorporation, Extended Data Fig. $\mathbf{2 g}$ ). Importantly, loss of RBPMS did not alter the level of pluripotency markers or hESC homeostasis (Fig. 2f, g, Extended Data Fig. $\mathbf{2 m}$ ) over a period of $>10$ passages. Together, the dramatic reduction in polysome occupancy and protein synthesis reveal a central role of RBPMS in regulating translation in hESCs.

\section{RBPMS is essential for mesoderm commitment and subsequent cardiogenesis}

The blueprint of the body plan is established during lineage commitment of pluripotent stem cells, a process that relies on the efficient rewiring of the proteome to support rapid cell identity changes upon instructive morphogen signaling. Because RBPMS loss abrogated protein synthesis in hESCs, we reasoned that its loss would hamper cell-fate decisions enabling lineage commitment. To investigate the role of RBPMS in this process, we used a defined and directed differentiation method towards the three primary germ layers: ectoderm, mesoderm, and endoderm, recapitulating molecularly the early embryonic cell fate decisions, as reported previously (Fig. 2 h) ${ }^{11}$.

Loss of RBPMS severely and specifically inhibited mesoderm commitment without affecting endoderm and ectoderm differentiation (Fig. 2i, Extended Data Fig. 2i-I). RBPMS loss abolished the ability of hESCs to effectively activate the key mesoderm, as well as cardiac mesoderm, commitment factors BRACHYURY (TBX-T) and MESP1 (Fig. 2i-k) ${ }^{47-49}$. In addiiton, upon mesoderm induction, RBPMS-KO cells still expressed pluripotency factors aberrantly (Extended Data Fig. 2p). Comparative transcriptome analyses of RBPMS-KO and WT cells at the state of pluripotency and upon mesoderm induction further confirmed their inability to effectively induce the mesoderm gene expression program (Fig. 2l, Extended Data Fig.

2n, Source Data Fig. 2). Of note, as we previously observed at the state of pluripotency, the transcriptional 
signature of core pluripotency factors was comparable between RBPMS-KO and WT cells (Extended Data Fig. 2m, Source Data Fig. 2). Strikingly, functional annotation of differentially-expressed genes revealed that morphogen signaling central to mesoderm commitment, including WNT and key pathways involved in mesoderm differentiation, failed to be activated in RBPMS-KO cells upon mesoderm induction, whereas genes involved in pluripotency and ectoderm differentiation were aberrantly upregulated (Fig. 2m, Extended Data Fig. 2o, p, Source Data Fig. 2). Expression of lineage marker undergoes rapid dynamics during early stages of germ layer commitment. To confirm that mesoderm commitment defects due to RBPMS loss were not a result of disrupted timing, we analyzed the expression dynamics of key mesoderm markers at close intervals. In agreement with our previous observations, mesodermal markers as well as WNT signaling mediators failed to activate in RBPMS-KO cells over the course of $24 \mathrm{~h}$ of mesoderm induction (Extended Data Fig. 3a). This goes hand in hand with substantially reduced TBX-T (a master regulator of mesoderm commitment) in RBPMS-KO hESCs upon mesoderm induction (Fig. 2k).

Last, we tested whether the impaired differentiation of RBPMS-KO hESCs to mesodermal lineage has functional consequences, detrimentally affecting terminal fate choices. In this regard, we chose defined differentiation to cardiomyocytes since it is a robust, high-efficiency method allowing nearsynchronous differentiation to a functional terminal fate ${ }^{50}$. Strikingly, RBPMS-KO cells failed to produce cardiomyocytes in contrast to WT cells that constantly yielded homogenous populations of cardiomyocytes (Fig. 2n). Additionally, RBPMS-KO cells failed to produce cardiomyocytes across the "cardiac corridor" (Extended Data Fig. 2q), which is a BMP/WNT concentration grid for testing the ability of pluripotent stem cells to give rise to cardiomyocytes ${ }^{50}$. Thus, we conclude that RBPMS is essential for accurate cell-fate decisions allowing mesoderm commitment and cardiac differentiation.

\section{RBPMS targets central regulators of mesoderm-specification and morphogenesis}

To comprehensively identify the network of mRNAs regulated by RBPMS in hESCs, we employed enhanced UV cross-linking and immunoprecipitation of ribonucleoprotein complex followed by massively parallel sequencing (eCLIP-seq) (Fig. 3a, Extended Data Fig. 4a, b $)^{51}$. Following removal of PCR duplicates and normalization relative to size-matched input controls from four independent replicates, we compiled transcriptome-wide, nucleotide-resolution and high confidence binding maps displaying $>80 \%$ overlap of target mRNAs between replicates (Fig. 3b, Extended Data Fig. 4c-e, Source Data Fig. 3). Only statistically significantly-enriched targets represented in all replicates were considered for further analysis. RBPMS was predominantly found bound to 3'UTRs of mRNAs (Fig. 3c, d, Source Data Fig 3) on a bipartite CAC 
motif (Fig. 3e, Extended Data Fig. 4f) in hESCs. Off note, RBPMS displayed substantially higher affinity for 3' UTR binding in comparison to other parts of the opern reading frame (Fig. 3c, d, Source Data Fig 3).

Functional annotation of 3'UTR-bound targets revealed that RBPMS binds mRNAs regulating gastrulation, tissue morphogenesis, as well as signal transduction - particularly WNT signaling, which are all essential for successful cell fate commitment during gastrulation ${ }^{8,52,53}$ (Fig. 3f, Source Data Fig. 3). 3'UTR targets of RBPMS are factors operating in a variety of cellular compartments (Extended Data Fig. $\mathbf{4 g}$ ). A curated set of such 3'UTR targets, selected for high signal-over-input enrichment and grouped on the basis of their molecular and developmental functions illustrates how RBPMS targets a broad network of mRNAs encoding crucial regulators of morphogen signal transduction and cell fate commitment that enables mesoderm development (Fig. 4g, h).

\section{Mesoderm specification is translationally specialized by RBPMS}

The sequestration of RBPMS on ribosomal complexes in hESCs, the severe inhibition of translation along with the specific mesoderm commitment defect in RBPMS-KO, and the direct binding of RBPMS to the 3'UTR of transcripts encoding key mesoderm-instructive developmental regulators led us to hypothesize that, in hESCs, RBPMS is a vital TSF essential for mesoderm specification. To test this hypothesis, we systematically evaluated the transcriptome-wide occupancy of ribosomal complexes in RBPMS-KOs compared to isogenic WTs by employing translation state RNA sequencing (TS-Seq). To this end, transcripts associated with ribosomal complexes (the 40S, 80S, light, and heavy polysomes) were isolated after ribosome fractionation, enriched for poly-adenylated transcripts and subjected to transcriptome sequencing, in parallel with total RNA from RBPMS-KO hESCs and corresponding isogenic WT (Extended Data Fig. 5a). To correct for technical variability and allow data normalization, two different sets of spikeins were added to each fraction of our three biological replicates, one after lysis and the other after polysome fractionation

The loss of RBPMS resulted in severe systems-wide translational inhibition, rendering the evaluation of changes in ribosome occupancy on specific mRNAs cumbersome (Fig. 2c-e). Therefore, to identify meaningful changes in ribosome occupancy, after normalization with dual spike-in controls, a two-step regression-based clustering approach was used. This approach allowed the identification of statistically significant transcript clusters that were substantially different in their ribosome occupancy pattern across the translational platforms in RBPMS-KO in comparison to isogenic WT hESCs.

We identified 8 mRNA clusters, harboring $>5500$ mRNAs, exhibiting significant differences in the pattern of ribosome occupancy between RBPMS-KO and WT hESCs. Importantly, six clusters harboring 
>3800 mRNAs were severely depleted from ribosomal complexes upon loss of RBPMS, while two clusters showed enrichment (Fig. 3i, Source Data Fig. 3). Translationally repressed genes were crucial for developmental cell fate commitment and embryogenesis, while translationally activated genes were involved in unrelated processes (Fig. 3j, Extended Data Fig. 5d, Source Data Fig. 3). Critically, integrative analysis of transcriptomics and TS-Seq data revealed that the transcripts only changing transcriptionally in the absence of RBPMS are not directly implicated in morphogen signaling or mesoderm/cardiacmesoderm development, the major processes regulated by RBPMS (Extended Data Fig. 5b, Source Data Fig. 3).

Our eCLIP-Seq data revealed that RBPMS predominately binds 3'UTRs of its target mRNAs. To investigate the direct connection between RBPMS binding and the translational status of its target mRNAs, we next investigated the ribosome occupancy on direct RBPMS targets. The majority of the RBPMS targets where it binds to the 3'UTR, were strongly depleted from ribosomal fractions in RBPMS-KOs, as illustrated by metagene analysis integrating eCLIP-peaks with ribosome occupancy of the respective transcripts (Fig. 3k, Source Data Fig. 3). These data reveal that RBPMS binding to the 3'UTR of its target mRNAs determines their ribosome occupancy and by extension their translational status.

The translationally inhibited 3'UTR targets of RBPMS are central regulators of mesoderm specification, cell-fate commitment during development, and importantly, morphogen signaling including WNT signaling (Fig. 3l). Since morphogen signaling, particularly by WNT, BMP/NODAL, and FGF defines mesoderm commitment from pluripotency, we then investigated the ribosome occupancy on genes known to be regulating these processes ${ }^{6,12}$. Strikingly, a majority of the components of WNT signal transduction along with those of BMP/NODAL and FGF signaling were severely depleted from active translational compartments upon RBPMS-KO, revealing the direct role for RBPMS-mediated translational specialization in embryonic cell fate decisions (Fig. 3m, Extended Data Fig. 5c, Source Data Fig. 3). The levels of active beta-catenin, a central WNT signaling component, were substantially low upon RBPMS loss (Fig. 3n), further confirming its direct role in controlling mesoderm specification. However, RBPMS does not affect transcript stability in general (assessed for a selection of pluripotency factors and direct RBPMS 3'UTR targets involved in WNT signaling, following actinomycin-D treatment to inhibit transcription) (Extended Data Fig. 5i). RBPMS was recently suggested to regulate splicing in smooth muscle cells ${ }^{54}$, extrapolated from targets identified by overexpression of RBPMS in HEK293T cells which naturally do not express RBPMS ${ }^{55}$. A cursory analysis revealed minimal overlap between the reported targets in HEK293-T cells and the targets we identified in hESCs, indicating cell type specificity of its endogenous target. Additionally, we find no evidence for splicing being the primary RBPMS function in hESCs. The loss of 
RBPMS severely impairs the ribosome occupancy of crucial developmental genes involved in the control of early embryonic fate determination at the state of pluripotency (illustrated by the translational status of the curated list of key developmental genes affected by its loss, including key 3'UTR targets; Extended Data Fig. 5e), revealing a central role for RBPMS in the translational specialization of cell fate commitment from pluripotency. Importantly, the depletion of ribosomal complexes globally as well as from RBPMS 3'UTR targets results in significant reduction in the abundance of corresponding proteins, confirmed by total proteome analysis using LC-MS comparing WT and RBPMS-KO hESCs (Extended Data Fig. 5f-h). Together, our data confirm that the abundance of the regulators of cell-fate decisions, including components of morphogen signal transduction crucial for mesoderm commitment (Extended Data Fig. $\mathbf{5 g}, \mathbf{h}$ ) are balanced in the state of pluripotency by RBPMS-mediated translation specialization, thereby poising future lineage choices.

\section{RBPMS is essential for the canonical composition of translation initiation complexes in hESCs}

RBPMS loss caused a systems-wide inhibition of translation, as well as specific depletion of ribosomes from its direct targets. This suggested that the role of RBPMS in translation could be two pronged (i) as a general regulator of translation in hESCs, and (ii) as a regulator of selective translation of mesoderm instructive cell-fate regulators. For an unbiased investigation of its associated factors, we performed direct immunoprecipitation of RBPMS followed by mass spectrometry from hESCs after prolonged RNase I treatment to avoid indirect RNA-mediated associations (Extended Data Fig. 6a, 6b). RBPMS specifically co-purified with canonical regulators of translation initiation including elF3 complex components, elF5A, as well as elF4G, along with multiple ribosomal subunits, suggesting a role for RBPMS in canonical organization of translation initiation complexes in hESCs (Fig. 4a, Extended Data Fig. 6c). Accordingly, we interrogated the dynamics of key factors influencing translation initiation on ribosomal platforms upon RBPMS loss. First, we specifically interrogated the enrichment of key initiation factor elF4G (involved in cross-talk between 435 preinitiation complex and elF4F complex), and poly(A)-binding protein PABP. Both displayed comparable levels indicating that RBPMS loss does not influence the predisposition of mRNAs to be translated (Fig. $\mathbf{4 b}, \mathbf{c}$ ). The slight reduction of elF4G from polysomal fractions in RBPMS-KO is indicative of translation inhibition. However, a key component of the 435 preinitiation complex and a regulatory hub for global translation, elF $2 A^{56}$, showed aberrant retention across ribosomal fractions following RBPMS loss (Fig. 4b, c). Additionally, two vital elF3 complex components, elF3E (involved in selective translation) $)^{57,58}$ and elF3H (previously reported to be involved in selective translation during embryonic development $)^{59}$ were aberrantly retained in polysomal fractions and substantially depleted 
from the $40 \mathrm{~S}$ complex in RBPMS-KO cells, respectively (Fig. 4b, c). Strikingly, elF5A, essential for proper elongation, for error resolution at ribosomal pause sites and for termination ${ }^{60}$, was sequestered into the 40S ribosomal fraction in the absence of RBPMS (Fig. 4b, c). Notably, the total levels of these factors do not reflect the change in their pattern of distribution in ribosomal fractions in RBPMS-KO hESCs (Extended

Data Fig. 6d). Together our data reveal that RBPMS loss results in aberrant retention of translation initiation factors on ribosomal complexes in hESCs.

Next, we asked whether binding by RBPMS to the 3'UTR of model mRNAs suffices for controlling their translation in hESCs. To this end, we generated a set of dual luciferase-based reporter constructs with RBPMS binding sites in the 3'UTR of SFRP1 or ACTB mRNAs. Loss of RBPMS lead to a significant decrease in luciferase activity only in SFRP1- 3'UTR fusions (with chicken ACTB and luciferase-only controls remaining unaffected compared to WT cells; Fig. 4d). Since luciferase-based reporter assays are end-state readouts, we used time-lapse microscopy of fluorescent reporters harboring RBPMS binding sites at the 3'UTR along with indicated controls to independently measure the linear influence of RBPMS on reporter levels (and by end read out at $24 \mathrm{~h}$ post-transfection). We observed significant increase in the translation efficiency in the presence of RBPMS only when its binding sites were present in the 3'UTR (Extended Data Fig. 6f, g). Together, our data confirm the direct role of RBPMS as a TSF in hESCs.

Finally, to confirm the role of RBPMS as a translation specialization factor central to mesoderm specification and to rule out discrepancies in our findings caused by genome engineering "off target" effects in RBPMS-KO hESCs, we knocked-in an inducible copy of RBPMS using PiggyBac transposon-based genomic insertion (Fig. 4e). Timely re-expression of the most prominent cytosolic isoform (the lowest band in RBPMS immunoblot; Fig. 1I) of RBPMS in RBPMS-KO cells fully restored both ribosome occupancy defects and protein synthesis, including the protein levels of SFRP1, one of the RBPMS 3'UTR targets, and a component of WNT signaling (Fig. 4g, h, Extended Data Fig. 6e) ${ }^{61}$. Importantly, re-expression of RBPMS restored mesoderm commitment capacity, reinstating the ability of RBPMS-KO cells to generate terminally differentiated cardiomyocytes (Fig. 4i). Taken together, our data reveal that the competence of hESCs for mesoderm specification is translationally specialized by RBPMS through the selective translation of developmental factors such as central morphogen signaling components, thereby poising future lineage choices.

In summary, we propose a model by which the state of pluripotency is translationally poised for differentiation into future lineages via the selective translation of the regulators of embryonic cell-fate. This is exemplified by translational specialization by RBPMS, which unlocks the mesoderm commitment potential of hESCs via selective translation of key developmental regulators, including morphogen and 
signal transduction components. The combination of BMP/WNT signaling faced by hESCs at the state of pluripotency predetermines their mesodermal and cardiac fate commitment potential in vitro ${ }^{6,62,63}$, while cells exiting the primitive streak in vivo are exposed to a specific ratio of WNT to BMP signaling gradient, which defines the mesodermal lineages they will differentiate into $8,52,53$. The regulation of this unique predisposition to terminal fate is currently unknown. Our work identified RBPMS as a translation specialization factor enabling mesoderm specification, based on which we propose an RBPMS-centric translational module specialized for mesoderm development. This is well supported by the ribosome foot printing studies from mesodermal cells in vivo ${ }^{26}$. Regulation of gene expression is paramount to developmental cell-fate transitions. In this regard, traditionally the focus has been mainly on delineating signaling pathways, transcriptional and epigenetic mechanisms contributing to developmental cell-fate decisions. We propose that TSFs associate with ribosomal platforms to regulate translation of cell-fate regulatory mRNAs in time and space, akin to how transcription factors program the transcriptional output of RNA Pol II, allowing embryonic cell fate decisions. Our catalog of translation machinery-associated proteins provides a rich resource for investigating additional translational specialization paradigms, and reveals a new regulatory space to be explored in the future. Collectively, we propose a pivotal role for translational specialization in sculpting cellular identity during early developmental lineage decisions.

\section{Acknowledgments}

The Kurian lab is supported by the NRW Stem Cell Network Independent Group Leader Grant (Grant No: 3681000801 and 2681101801, Else Kröner-Fresenius- Stiftung, Deutsche Forschungsgemeinschaft (DFG) and University of Cologne (Grant No: 3622801511). We also acknowledge the central facilities at CECAD and CMMC for technical assistance. We thank Prof. Gene Yeo (UCSD) and Asst. Prof Eric Van Nostrand (Baylor College) for sharing the eCLIP technology. We would like to thank Dr. Janine Altmueller, CCGCologne, and Dr. Jan-Wilm Lackmann, CECAD Mass spec facility for advice and assistance.

\section{Author contributions}

L.K. conceived and supervised the projects. L.K and D.B designed all the experiments. G.A, and D.B conducted all the computational analysis. D. B and K. K performed the experiments. A.P and H.B assisted in various stages of the project. L.K and D.B analyzed and interpreted the data. L.K and D.B. drafted all illustrations, with assistance from G.A. L.K prepared the manuscript. 


\section{Declaration of interests}

The authors declare no competing interests.

Supplementary Information is available for this paper. Correspondence and requests for materials should be addressed to: leo.kurian@uni-koeln.de

\section{References}

1 Schwanhausser, B. et al. Global quantification of mammalian gene expression control. Nature 473, 337-342, doi:10.1038/nature10098 (2011).

2 Teixeira, F. K. \& Lehmann, R. Translational Control during Developmental Transitions. Cold Spring Harbor perspectives in biology 11, doi:10.1101/cshperspect.a032987 (2019).

3 Genuth, N. R. \& Barna, M. Heterogeneity and specialized functions of translation machinery: from genes to organisms. Nature reviews. Genetics 19, 431-452, doi:10.1038/s41576-018-0008-z (2018).

4 Sampath, P. et al. A hierarchical network controls protein translation during murine embryonic stem cell self-renewal and differentiation. Cell stem cell 2, 448-460, doi:10.1016/j.stem.2008.03.013 (2008).

5 Ingolia, N. T., Lareau, L. F. \& Weissman, J. S. Ribosome profiling of mouse embryonic stem cells reveals the complexity and dynamics of mammalian proteomes. Cell 147, 789-802, doi:10.1016/j.cell.2011.10.002 (2011).

6 Loh, K. M. et al. Mapping the Pairwise Choices Leading from Pluripotency to Human Bone, Heart, and Other Mesoderm Cell Types. Cell 166, 451-467, doi:10.1016/j.cell.2016.06.011 (2016).

7 Xiang, L. et al. A developmental landscape of 3D-cultured human pre-gastrulation embryos. Nature 577, 537-542, doi:10.1038/s41586-019-1875-y (2020).

8 Peng, G. et al. Molecular architecture of lineage allocation and tissue organization in early mouse embryo. Nature 572, 528-532, doi:10.1038/s41586-019-1469-8 (2019).

9 Newport, J. \& Kirschner, M. A major developmental transition in early Xenopus embryos: II. Control of the onset of transcription. Cell 30, 687-696, doi:10.1016/0092-8674(82)90273-2 (1982).

10 Newport, J. \& Kirschner, M. A major developmental transition in early Xenopus embryos: I. characterization and timing of cellular changes at the midblastula stage. Cell 30, 675-686, doi:10.1016/0092-8674(82)90272-0 (1982).

11 Frank, S. et al. yylncT Defines a Class of Divergently Transcribed IncRNAs and Safeguards the Tmediated Mesodermal Commitment of Human PSCs. Cell stem cell 24, 318-327 e318, doi:10.1016/j.stem.2018.11.005 (2019).

12 Brade, T., Pane, L. S., Moretti, A., Chien, K. R. \& Laugwitz, K. L. Embryonic heart progenitors and cardiogenesis. Cold Spring Harbor perspectives in medicine 3, a013847, doi:10.1101/cshperspect.a013847 (2013).

13 Stadhouders, R., Filion, G. J. \& Graf, T. Transcription factors and 3D genome conformation in cell-fate decisions. Nature 569, 345-354, doi:10.1038/s41586-019-1182-7 (2019).

14 Alberio, R. Regulation of Cell Fate Decisions in Early Mammalian Embryos. Annual review of animal biosciences 8, 377-393, doi:10.1146/annurev-animal-021419-083841 (2020). 
Kristensen, A. R., Gsponer, J. \& Foster, L. J. Protein synthesis rate is the predominant regulator of protein expression during differentiation. Molecular systems biology 9,689 , doi:10.1038/msb.2013.47 (2013).

16 Munoz, J. \& Heck, A. J. Quantitative proteome and phosphoproteome analysis of human pluripotent stem cells. Methods in molecular biology 767, 297-312, doi:10.1007/978-1-61779201-4_22 (2011).

17 Wolin, S. L. \& Walter, P. Ribosome pausing and stacking during translation of a eukaryotic mRNA. The EMBO journal 7, 3559-3569 (1988).

18 Munoz, J. L., Deyhimi, F. \& Coles, J. A. Silanization of glass in the making of ion-sensitive microelectrodes. Journal of neuroscience methods 8, 231-247, doi:10.1016/01650270(83)90037-7 (1983).

19 Arnheim, N. \& Southern, E. M. Heterogeneity of the ribosomal genes in mice and men. Cell 11, 363-370, doi:10.1016/0092-8674(77)90053-8 (1977).

20 Harding, H. P. et al. An integrated stress response regulates amino acid metabolism and resistance to oxidative stress. Molecular cell 11, 619-633, doi:10.1016/s1097-2765(03)00105-9 (2003).

21 Shi, Z. et al. Heterogeneous Ribosomes Preferentially Translate Distinct Subpools of mRNAs Genome-wide. Molecular cell 67, 71-83 e77, doi:10.1016/j.molcel.2017.05.021 (2017).

22 Lee, M. T., Bonneau, A. R. \& Giraldez, A. J. Zygotic genome activation during the maternal-tozygotic transition. Annual review of cell and developmental biology 30, 581-613, doi:10.1146/annurev-cellbio-100913-013027 (2014).

23 Kong, J. \& Lasko, P. Translational control in cellular and developmental processes. Nature reviews. Genetics 13, 383-394, doi:10.1038/nrg3184 (2012).

24 Hausser, J., Mayo, A., Keren, L. \& Alon, U. Central dogma rates and the trade-off between precision and economy in gene expression. Nature communications 10, 68, doi:10.1038/s41467018-07391-8 (2019).

25 Lamper, A. M., Fleming, R. H., Ladd, K. M. \& Lee, A. S. Y. A phosphorylation-regulated elF3d translation switch mediates cellular adaptation to metabolic stress. Science 370, 853-856, doi:10.1126/science.abb0993 (2020).

26 Fujii, K., Shi, Z., Zhulyn, O., Denans, N. \& Barna, M. Pervasive translational regulation of the cell signalling circuitry underlies mammalian development. Nature communications 8, 14443, doi:10.1038/ncomms14443 (2017).

27 Leppek, K. et al. Gene- and Species-Specific Hox mRNA Translation by Ribosome Expansion Segments. Molecular cell 80, 980-995 e913, doi:10.1016/j.molcel.2020.10.023 (2020).

28 Leppek, K., Das, R. \& Barna, M. Functional 5' UTR mRNA structures in eukaryotic translation regulation and how to find them. Nature reviews. Molecular cell biology 19, 158-174, doi:10.1038/nrm.2017.103 (2018).

29 Xue, S. et al. RNA regulons in Hox 5' UTRs confer ribosome specificity to gene regulation. Nature 517, 33-38, doi:10.1038/nature14010 (2015).

30 Simsek, D. et al. The Mammalian Ribo-interactome Reveals Ribosome Functional Diversity and Heterogeneity. Cell 169, 1051-1065 e1018, doi:10.1016/j.cell.2017.05.022 (2017).

31 Mauro, V. P. \& Edelman, G. M. The ribosome filter hypothesis. Proceedings of the National Academy of Sciences of the United States of America 99, 12031-12036, doi:10.1073/pnas.192442499 (2002).

32 Shi, Z. \& Barna, M. Translating the genome in time and space: specialized ribosomes, RNA regulons, and RNA-binding proteins. Annual review of cell and developmental biology 31, 31-54, doi:10.1146/annurev-cellbio-100814-125346 (2015). 
Iserman, C. et al. Condensation of Ded1p Promotes a Translational Switch from Housekeeping to Stress Protein Production. Cell 181, 818-831 e819, doi:10.1016/j.cell.2020.04.009 (2020).

34 Markmiller, S. et al. Context-Dependent and Disease-Specific Diversity in Protein Interactions within Stress Granules. Cell 172, 590-604 e513, doi:10.1016/j.cell.2017.12.032 (2018). Kedersha, N. \& Anderson, P. Stress granules: sites of mRNA triage that regulate mRNA stability and translatability. Biochemical Society transactions 30, 963-969, doi:10.1042/bst0300963 (2002). link the phosphorylation of elF-2 alpha to the assembly of mammalian stress granules. The Journal of cell biology 147, 1431-1442, doi:10.1083/jcb.147.7.1431 (1999).

37 Brannan, K. W. et al. SONAR Discovers RNA-Binding Proteins from Analysis of Large-Scale Protein-Protein Interactomes. Molecular cell 64, 282-293, doi:10.1016/j.molcel.2016.09.003 (2016).

38 Kwon, S. C. et al. The RNA-binding protein repertoire of embryonic stem cells. Nature structural \& molecular biology 20, 1122-1130, doi:10.1038/nsmb.2638 (2013).

39 Hentze, M. W., Castello, A., Schwarzl, T. \& Preiss, T. A brave new world of RNA-binding proteins. Nature reviews. Molecular cell biology 19, 327-341, doi:10.1038/nrm.2017.130 (2018).

40 Kozel, C. et al. Overexpression of elF5 or its protein mimic 5MP perturbs elF2 function and induces ATF4 translation through delayed re-initiation. Nucleic acids research 44, 8704-8713, doi:10.1093/nar/gkw559 (2016).

41 Negrutskii, B. S. \& Deutscher, M. P. Channeling of aminoacyl-tRNA for protein synthesis in vivo. Proceedings of the National Academy of Sciences of the United States of America 88, 4991-4995, doi:10.1073/pnas.88.11.4991 (1991).

42 Bianco, C. \& Mohr, I. Ribosome biogenesis restricts innate immune responses to virus infection and DNA. eLife 8, doi:10.7554/eLife.49551 (2019).

43 Brecht, R. M. et al. Nucleolar localization of RAG1 modulates V(D)J recombination activity. Proceedings of the National Academy of Sciences of the United States of America 117, 4300 4309, doi:10.1073/pnas.1920021117 (2020).

44 Gerber, W. V. et al. The RNA-binding protein gene, hermes, is expressed at high levels in the developing heart. Mechanisms of development 80, 77-86, doi:10.1016/s0925-4773(98)00195-6 (1999).

45 Gerber, W. V., Vokes, S. A., Zearfoss, N. R. \& Krieg, P. A. A role for the RNA-binding protein, hermes, in the regulation of heart development. Developmental biology 247, 116-126, doi:10.1006/dbio.2002.0678 (2002).

46 Aguero, T. et al. Hermes (Rbpms) is a Critical Component of RNP Complexes that Sequester Germline RNAs during Oogenesis. Journal of developmental biology 4, doi:10.3390/jdb4010002 (2016).

47 Bondue, A. et al. Mesp1 acts as a master regulator of multipotent cardiovascular progenitor specification. Cell stem cell 3, 69-84, doi:10.1016/j.stem.2008.06.009 (2008).

48 Tosic, J. et al. Eomes and Brachyury control pluripotency exit and germ-layer segregation by changing the chromatin state. Nature cell biology 21, 1518-1531, doi:10.1038/s41556-019-04231 (2019).

49 Perea-Gomez, A. et al. Initiation of gastrulation in the mouse embryo is preceded by an apparent shift in the orientation of the anterior-posterior axis. Current biology : CB 14, 197-207, doi:10.1016/j.cub.2004.01.030 (2004).

50 Rao, J. et al. Stepwise Clearance of Repressive Roadblocks Drives Cardiac Induction in Human ESCs. Cell stem cell 18, 554-556, doi:10.1016/j.stem.2016.03.008 (2016). 
51 Van Nostrand, E. L. et al. Robust transcriptome-wide discovery of RNA-binding protein binding sites with enhanced CLIP (eCLIP). Nature methods 13, 508-514, doi:10.1038/nmeth.3810 (2016).

52 Bisson, J. A., Mills, B., Paul Helt, J. C., Zwaka, T. P. \& Cohen, E. D. Wnt5a and Wnt11 inhibit the canonical Wnt pathway and promote cardiac progenitor development via the Caspasedependent degradation of AKT. Developmental biology 398, 80-96, doi:10.1016/j.ydbio.2014.11.015 (2015).

53 Marvin, M. J., Di Rocco, G., Gardiner, A., Bush, S. M. \& Lassar, A. B. Inhibition of Wnt activity induces heart formation from posterior mesoderm. Genes \& development 15, 316-327, doi:10.1101/gad.855501 (2001).

54 Nakagaki-Silva, E. E. et al. Identification of RBPMS as a mammalian smooth muscle master splicing regulator via proximity of its gene with super-enhancers. eLife $\mathbf{8}$, doi:10.7554/eLife.46327 (2019).

55 Farazi, T. A. et al. Identification of the RNA recognition element of the RBPMS family of RNAbinding proteins and their transcriptome-wide mRNA targets. Rna 20, 1090-1102, doi:10.1261/rna.045005.114 (2014).

56 Wek, R. C. Role of elF2alpha Kinases in Translational Control and Adaptation to Cellular Stress. Cold Spring Harbor perspectives in biology 10, doi:10.1101/cshperspect.a032870 (2018).

57 Lin, Y. et al. elF3 Associates with 80S Ribosomes to Promote Translation Elongation, Mitochondrial Homeostasis, and Muscle Health. Molecular cell 79, 575-587 e577, doi:10.1016/j.molcel.2020.06.003 (2020).

58 Shah, M. et al. A Transcript-Specific elF3 Complex Mediates Global Translational Control of Energy Metabolism. Cell reports 16, 1891-1902, doi:10.1016/j.celrep.2016.07.006 (2016).

59 Choudhuri, A., Maitra, U. \& Evans, T. Translation initiation factor elF3h targets specific transcripts to polysomes during embryogenesis. Proceedings of the National Academy of Sciences of the United States of America 110, 9818-9823, doi:10.1073/pnas.1302934110 (2013). Schuller, A. P., Wu, C. C., Dever, T. E., Buskirk, A. R. \& Green, R. elF5A Functions Globally in Translation Elongation and Termination. Molecular cell 66, 194-205 e195, doi:10.1016/j.molcel.2017.03.003 (2017).

61 Liang, C. J. et al. SFRPs Are Biphasic Modulators of Wnt-Signaling-Elicited Cancer Stem Cell Properties beyond Extracellular Control. Cell reports 28, 1511-1525 e1515, doi:10.1016/j.celrep.2019.07.023 (2019).

62 Rao, J. et al. Stepwise Clearance of Repressive Roadblocks Drives Cardiac Induction in Human ESCs. Cell stem cell 18, 341-353, doi:10.1016/j.stem.2015.11.019 (2016).

63 Kempf, H. et al. Bulk cell density and Wnt/TGFbeta signalling regulate mesendodermal patterning of human pluripotent stem cells. Nature communications 7, 13602, doi:10.1038/ncomms13602 (2016). 


\section{Figure legends}

Fig. 1: Systematic analysis of proteins residing on ribosomal complexes identifies RBPMS as candidate translation specialization factor in hESCs

(a) A schematic outline of the underlying strategy employed for translation state mass spectrometry (TS$\mathrm{MS})$.

(b) RNA binding proteins are the largest class of protein on ribosomal platforms in hESCs. $n=$ total number for proteins/ribosomal complex, (only proteins detected in all three biological replicates above $\log _{2}$ LFQ $\geq 25$, FDR $\leq 0.1$ were considered). (LFQ: label-free quantification; LC-MS: liquid chromatography mass-spectrometry)

(c) Gene Ontology-based functional enrichment analysis for differentially enriched proteins on ribosomal complexes grouped under overarching functional categories ( $p$-value $\leq 0.001$ ). (FC: fold change)

(d) Dynamics of RNA binding proteins residing on ribosomal complexes in steady state conditions and upon transient translational challenge $(n=600)$.

(e) Differential enrichment-based ranking of RBPs on ribosomal complexes between steady state and upon translational challenge computed by Hotelling's two-tailed T2, top 5 enriched RBPs in the inlet (Hotelling's T2 values for ranking translation machinery-associated proteins using MEBA implemented using MetaboAnalyst).

(f), (g) RBPMS resides predominantly on $40 \mathrm{~S}$ complex in hESCs in steady state conditions and migrates to polysomal complexes upon translational challenge, evaluated by mass spectrometry (line graph) and by western blotting. RPL7A, RPS6, and G3BP1 serve as controls.

(h) RBPMS is a predominantly cytosolic protein, evaluated by Western blot analysis upon nuclear/cytosolic fractionation, G3BP1, and TUBA1B cytosolic control, LAMINB1 nuclear control. (i) Residence of RBPMS on ribosomal complexes evaluated and confirmed by its characteristic association with the indicated complexes upon treatment with respective translation inhibitors, evaluated by polysome fractionation. Protein levels of RBPMS and the indicated controls on ribosomal fractions were detected by Western blotting.

Error bars represent \pm SEM; $p$-values calculated using Student's t-test, * $\leq 0.05$ )

See also Extended Data Fig. 1. 


\section{Fig. 2: RBPMS is essential for translational homeostasis in hESCs and its loss abrogates mesoderm specification}

(a) Schematic representation of RBPMS locus in humans and the CRISPR/Cas9-based targeting strategy used to generate homozygous RBPMS-KO, confirmed at the (b) at the protein level.

(c) Loss of RBPMS impedes translation in hESCs, indicated by representative polysome profiles from sucrose gradient fractionation of RBPMS-KO hESCs compared to isogenic WTs along with quantification of the area under the indicated ribosomal fractions on the right.

Protein synthesis is inhibited in RBPMS-KO hESCs. De novo protein synthesis is evaluated by measuring puromycin incorporation on nascent proteins using (d) anti-puromycin antibody by western blotting and (e) by measuring uptake of O-Propargyl-puromycin (OPP) quantifications on the right.

(f) RBPMS-loss does not affect pluripotency. Representative images of WT and RBPMS-KO hESCs stained for OCT4, SOX2 and NANOG: Bar graph shows normalized expression levels of indicated pluripotency markers.

(g) RT-qPCR for pluripotency markers OCT4, NANOG, and SOX2. Bar graphs show relative fold change to WT hESCs normalized to RPL37A.

(h) Schematic of lineage differentiation approaches used to determine the competence of RBPMS-KO hESCs to undergo germline commitment.

RBPMS loss abrogates the ability of hESCs to commit to mesoderm, evaluated by (i) immunofluorescence imaging for MESP1 (central mesoderm specifier), (j) RT-qPCR for MESP1 and TBX-T (master regulator for mesoderm specification and (k) by western blot analysis for TBX-T.

(i) Representative images of MESP1 staining upon mesoderm induction of RBPMS-KOs hESCs for indicated mesodermal markers, quantification on the right. Impaired mesoderm commitment was confirmed by RT-qPCR (j) highlighting reduced activation of mesoderm markers TBX-T and MESP1 RTqPCR for mesoderm markers TBX-T and MESP1, (k) as well as by western blotting for TBX-T.

(I) Heatmap shows normalized expression levels of indicated mesoderm markers

(m) Gene Ontology-based functional enrichment analysis for differentially expressed genes in WT mesoderm and RBPMS-KO mesoderm (significance level indicated by color code).

(n) Loss of RBPMS detrimentally affects terminal fate choices of hESCs indicated by defined differentiation to cardiomyocytes. Representative images of indicated markers upon cardiac differentiation of WT hESCS compared to RBPMS-KO hESCs.

Error bars represent \pm SEM; $p$-values calculated using Student's t-test ( $p$-values : n.s. $>0.05,{ }^{*} \leq 0.05$, $* * \leq 0.01, * * * \leq 0.001, * * * * \leq 0.0001, \mathrm{n}=3)$. 
See also Extended Data Fig. 2, 3

Fig. 3: Translational specialization of the regulators of mesoderm specification requires direct binding of RBPMS

(a) Schematic of the eCLIP-seq approach employed to faithfully generate an unbiased transcriptomewide direct binding map for RBPMS at single-nucleotide resolution in hESCs.

(b) Biological quadruplicates of eCLIP show at least $80 \%$ overlap between each other, pie charts show the correlation of statistically significant uniquely mapped reads for each replicate over SMInput.

(c) RBPMS binds predominantly the 3'UTR of its direct target transcripts, demonstrated here by the distribution of the significantly enriched eCLIP peaks against the paired SMInput (fold change $\geq 2 ; p$ value $\leq 0.05$ in all 4 replicates).

(d) Metagene plot visualizing the peak distribution of RBPMS over SMInput illustrating prominent 3'UTR binding.

(e) Top sequence motif significantly bound by RBPMS.

(f) 3'UTR targets of RBPMS regulate molecular processes central to mesoderm commitment including components of morphogen signal transduction network, depicted by significantly enriched GO terms.

(g) A curated set of RBPMS 3'UTR targets grouped based on their proven role in the indicated cellular, developmental and functional process, depicted as a heatmap of enrichment over SMInput.

(h) Representative read density tracks show read density for RBPMS across the gene body of SFRP1, a representative target.

(i) Global impact of the loss of RBPMS on ribosome occupancy in hESCs, revealed by two-step regression analysis of the mRNAs enriching on ribosomal fractions.

(j) Functional analysis of translationally repressed transcripts in RBPMS-KOs versus isogenic WT hESCs illustrated as a significantly enriched curated list of GO terms (significance levels indicated in the key on the right).

(k) Metagene plot indicating a 3'UTR binding bias for translationally repressed targets of RBPMS.

(I) Functional analysis of translationally repressed 3'UTR targets of RBPMS in RBPMS-KO hESCs compared to corresponding WT cells illustrated as a curated list of significantly enriched GO terms.

(m) Loss of RBPMS severely impairs ribosome occupancy on mRNAs encoding components of WNT, BMP, NODAL, and FGF signaling, vital mesoderm specifying signal transduction networks.

(n) Loss of RBPMS reduces active BETA CATENIN levels in hESCs as detected by Western blotting. 
Error bars represent \pm SEM; $p$-values calculated using Student's t-test $p$-values : n.s. $>0.05, * \leq 0.05$, $* * \leq 0.01, * * * \leq 0.001, * * * * \leq 0.0001, \mathrm{n}=3)$.

See also Extended Data Fig. 4, 5

\section{Fig. 4: RBPMS-loss results in aberrant retention of translation initiation factors on ribosomal} complexes

(a) Circos plot depicting the enrichment of indicated proteins upon immunoprecipitation of RBPMS after extended treatment with RNAse I over the IgG control. Log2 LFQ $\geq 25$ in all three biological replicates and $\log _{2}$ fold change over $\lg G \geq 2$, $p$-value $\leq 0.05$ ). ( $n=3$ unless otherwise indicated).

Error bars represent \pm SEM; $p$-values calculated using Student's t-test, $* 0.05$ )

(b), (c) Loss of RBPMS in hESCs results in impaired retention of translational initiation factors, involved in translational specialization, depicted by the distribution of the occupancy of indicated factors in ribosomal complexes, isolated by polysome fractionation. $(n=2)$.

(d) RBPMS enhances the translation of dual luciferase-based reporters specifically upon in the presence of its RNA binding motif. Indicated 3'UTRs were fused to firefly luciferase open reading frame, while renilla luciferase encoded by the same plasmid serves as control.

(e) Timely reconstitution of RBPMS in RBPMS-KO hESCs rescues (f) ribosome occupancy, (g) general protein synthesis, (h) translation defect of 3'UTR targets of RBPMS as well as (i) lineage commitment defects.

Error bars represent \pm SEM; $p$-values calculated using Student's t-test $p$-values : n.s. $>0.05,{ }^{*} \leq 0.05$, $* * \leq 0.01, * * * \leq 0.001, * * * * 0.0001, \mathrm{n}=3)$.

See also Extended Data Fig. 6 


\section{SUPPLEMENTARY FIGURE LEGENDS}

\section{Extended Data Fig. 1: Translational challenge rewires the proteome of ribosomal complexes in hESCs}

(a) Polysome traces from sucrose gradient fractionation of hESCs in steady state and upon transient translational challenge by $\mathrm{NaAsO}_{2}$.

(b) Transient translational challenge leads to significant depletion of polysomal fractions, shown by bar plots of the area under the indicated fractions from polysome traces.

(c) Transient translation challenge causes a substantial change in the total proteome of ribosomal complexes, indicated by the principal component analysis of the indicated fractions from TS-MS.

(d) Heatmap depicting the abundance of ribosomal proteins constituting small and large ribosomal subunits.

(e) The abundance of RNA binding proteins on polysomal fractions show a substantial increase upon transient translational stress. $\mathrm{n}=$ total number for protein/ribosomal complex, (only proteins detected in all three biological replicates above $\log _{2} \mathrm{LFQ} \geq 25, \mathrm{FDR} \leq 0.1$ were considered).

(f) Gene Ontology-based functional enrichment analysis for differentially enriched proteins on ribosomal platforms upon transient translational challenge ( $p$-value $\leq 0.001)$.

(g) The overlap between identified translation-associated factors with components of the indicated multiprotein complexes.

Extended Data Fig. 2: RBPMS loss does not affect cell cycle, energy metabolism, neuroectorderm or endoderm differentiation

(a) Sanger sequencing-based confirmation of genome editing in the generated RBPMS-KO hESCs.

(b) RBPMS mRNA levels in WT hESCS compared to RBPMS-KOs evaluated by qPCR. (c) Evaluation of the effect of cell cycle upon RBPMS loss in hESCs.

(d) Evaluation of mitochondrial distribution (mitofusin and Mitotracker staining) and (e) mitochondrial integrity (live Mitotracker intake) in RBPMS-KO and wildtype hESCs.

(f) Mitochondrial oxygen consumption rates (OCR; pmol $\mathrm{O}^{2} / \mathrm{min}$ ) in WT and RBPMS-deficient hESCs. Specific inhibitors used in the analysis are indicated.

(g) Representative micrographs depicting the nascent transcription in WT and RBPMS-KO hESCs, measured by EU (5-Ethynyl Uridine) labeling assay.

(h) De novo protein synthesis evaluated by measuring puromycin incorporation of nascent proteins using anti-puromycin antibody between WT and RBPMS-KO hESCs, evaluated by fluorescence microscopy. 
Loss of RBPMS does not affect (i) \& (j) neuroectoderm and (k) \& (I) endoderm commitment potential of hESCs, evaluated by immune fluorescence microscopy and qPCR of the indicated markers.

(m) Loss of RBPMS does not affect the expression of pluripotency markers in hESCs.

(n) Induction of mesoderm markers is affected upon RBPMS-KO.

(o) RBPMS-KO hESCS show aberrant expression of indicated endodermal and ectodermal markers upon mesoderm induction.

(p) Loss of RBPMS results in residual expression of indicated pluripotency markers upon mesoderm induction, evaluated by RT-qPCR analysis

(q) RBPMS-KO fail to differentiate to cardiomyocytes across the WNT-BMP gradient permissive for cardiac commitment, "cardiac corridor" as compared to WT hESCs.

Error bars represent $\pm S E M$; $p$-values calculated using Student's t-test ( $p$-values: n.s. $>0.5, * \leq 0.05,{ }^{* *} \leq 0.01$, $* * * \leq 0.001, * * * * \leq 0.0001, \mathrm{n}=3)$.

\section{Extended Data Fig. 3: RBPMS is essential for the timely activation of mesoderm specifiers}

(a) Kinetics of induction of mesodermal markers measured by $\mathrm{qPCR}$ at indicated intervals during mesoderm induction confirms mesoderm commitment defects upon RBPMS-KO in hESCs. Bar graphs represent gene expression kinetics of indicated markers.

Error bars represent \pm SEM; $p$-values calculated using Student's t-test (p-values: n.s.>0.5, $* 0.05, * * \leq 0.01$, $* * * \leq 0.001, * * * * \leq 0.0001, \mathrm{n}=3)$.

\section{Extended Data Fig. 4: eCLIP-seq maps direct RBPMS targets in hESCs}

(a) Representative Western blot-based validation of immunoprecipitation performed during eCLIP of RBPMS, TUBA1B serves as the control.

(b) Representative eCLIP libraries, illustrated as tape-station readouts.

(c) Uniquely mapped reads for all eCLIP samples.

(d) Read density of eCLIP-Seq data indicating fold enrichment over respective SMlinputs.

(e) Correlation of enriched eCLIP-peaks over SMInput between each replicate.

(f) Top sequence motif significantly bound by RBPMS along with peak distribution categorized based on binding of RBPMS on the mRNA coordinates for each replicate.

(g) Enriched GO terms (cellular component) found in RBPMS 3'UTR targets. 
Error bars represent \pm SEM; $p$-values calculated using Student's t-test (p-values: n.s. $>0.5, * \leq 0.05,{ }^{* *} \leq 0.01$, $* * * \leq 0.001, * * * * \leq 0.0001, \mathrm{n}=3)$.

Extended Data Fig. 5: The molecular and developmental function of RBPMS is primarily through its direct regulation of translation

(a) Schematic of the TS-Seq employed to evaluate the role of RBPMS globally as well as specifically on its direct targets.

(b) Functional annotation of genes affected transcriptionally alone upon loss of RBPMS does not suffice for the molecular and developmental defects due to the absence of RBPMS in hESCs.

(c) Dynamics of indicated mRNAs on the indicated ribosomal platforms, bound by RBPMS on the 3'UTR.

(d) Gene Ontology-based functional enrichment analysis for 3'UTR targets translationally activated in RBPMS-KO hESCs (significance level indicated by color code).

(e) Translational status of the curated list of key developmental genes upon loss of RBPMS, illustrated as heatmap depicting the ratio of occupancy of the indicated ribosomal complexes on transcripts between RBPMS-KO and WT hESCS.

(f) RBPMS loss results in systems wide reduction in protein abundance. Protein abundance evaluated by unbiased whole proteome analysis between WT hESCs and RBPMS-KOs ( $\mathrm{n}=3$, biological replicates).

(g) Reduction in ribosome occupancy on RBPMS 3'UTR targets results in substantial reduction in their protein abundance. Heat map depicting the protein levels of RBPMS 3'UTR targets evaluated by whole proteome analysis. Curated list of RBPMS 3'UTR targets are marked.

(h) Gene Ontology-based functional enrichment analysis RBPMS 3'UTR targets depleted at the protein level in RBPMS-KOs with respect to WT hESCs.

(i) Loss of RBPMS does not change the stability of indicated mRNAs, including RBPMS 3'UTR targets, revealed by time-course experiment upon Actinomycin B treatment.

Error bars represent \pm SEM; $p$-values calculated using Student's t-test $(n=3)$.

\section{Extended Data Fig. 5: Translational specialization of RBPMS-targets requires the presence of its binding} motif in the 3'UTR

(a) Coomassie blue-stained gels depicting the indicated eluates from RBPMS and IgG (control) pull-downs, respectively. Size separated proteins were pooled independently, isotope-labeled, and quantified by LCMS. 
(b) Volcano plot showing specific enrichment of proteins upon RBPMS pull-down w.r.t IgG control, in triplicates.

(c) Enrichment of ribosomal proteins and translation factors upon RBPMS pull down.

Error bars represent \pm SEM; $p$-values calculated using Student's t-test $(n=3)$.

(d) Total levels of the indicated proteins in RBPMS-KO hESCs compared to the corresponding WT.

(e) Levels of SFRP1 evaluated by western blot analysis in RBPMS-KO and WT hESCs, quantifications on the right.

(f) Schematic depicting the fluorescence-based translation reporter constructs used. Bar graphs depicting GFP mean fluorescence intensity (MFI) of the translation reporters in indicated conditions measured 24 hours post transfection shown in lower panel.

(g) RBPMS enhances the translation of fluorescence-based dual reporters specifically upon in the presence of its RNA binding motif. Indicated 3'UTRs were fused to GFP open reading frame, co-transfected with plasmids expressing RBPMS or mCherry in WT or RBPMS-KO hESCs and fluorescence was recorded continuously for $48 \mathrm{~h}$ by live microscopy.

Error bars represent \pm SEM; p-values calculated using Student's t-test (n.s. $>0.5,{ }^{*} \leq 0.05, * * \leq 0.01$, $* * * \leq 0.001, * * * * \leq 0.0001, \mathrm{n}=3)$. 


\section{hESCs}

$10 \%$

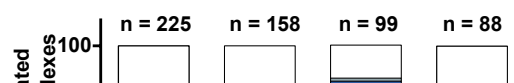

$40 S \backsim$

protein metabolism

protein metabolis

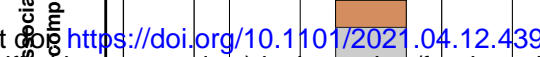

bioRxiv preprint

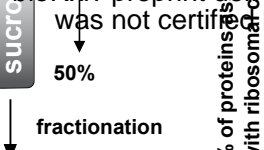

$40 S$ 80S polysomes
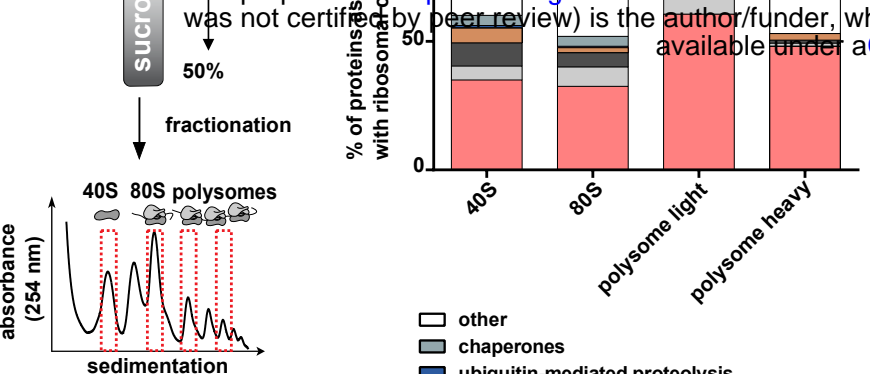

$\square$ chaperones

$\square$ ubiquitin-mediated proteolysis

LC-MS

$\square$ proteasome

$\square$ energy metabolism

$\square$ proteas

$\geq 25 \log _{2}$ LFQ

- in three biological replicates

- in at least one fraction

d
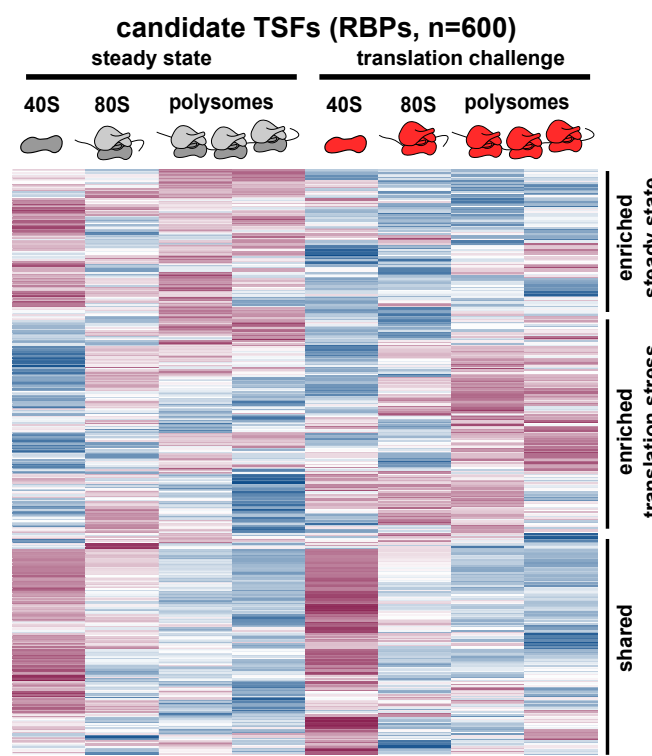

relative enrichment

$\begin{array}{lllll}-2 & -1 & 0 & 1 & 2\end{array}$

i
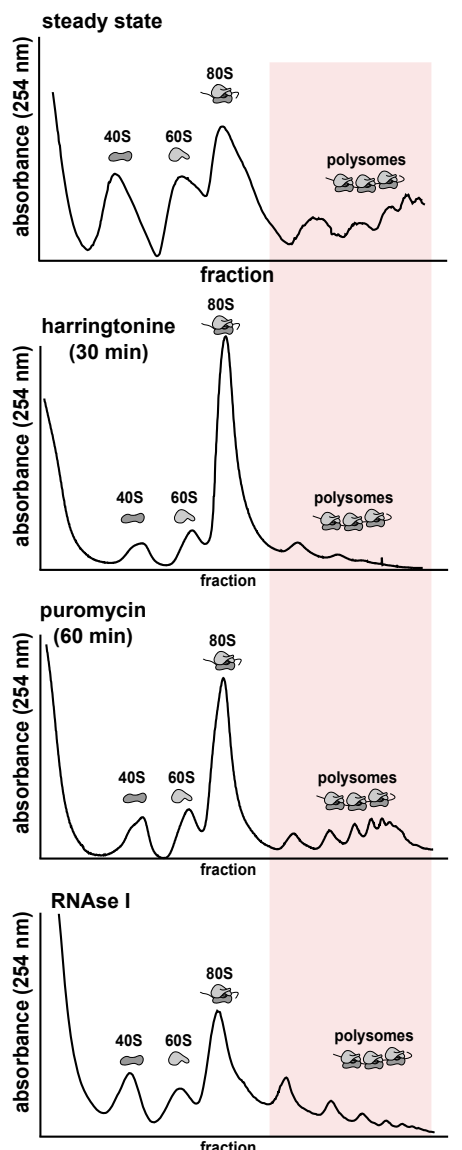

805 के cellular metabolism 000

polysome light cellular metabolism

The eopyright holder for this preprint (which The eopyright holder for this preprint (which
play the preprint in perpetuity. It is made

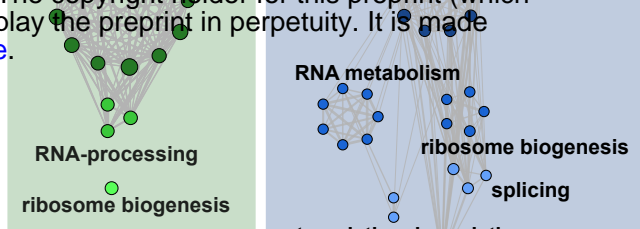

$n=158$
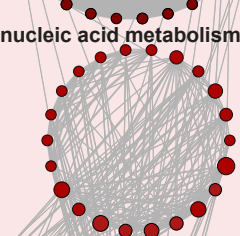

ribosome biogenesis
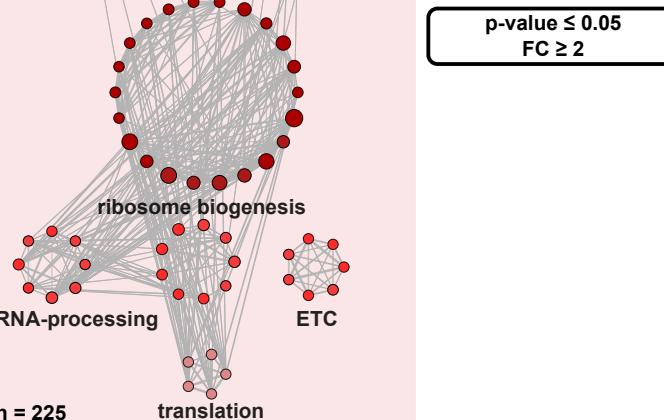

translational regulation

$\mathrm{n}=99$ gene expression

polysome heavy

cellular metabolism

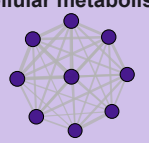

RNA transport

\section{f}
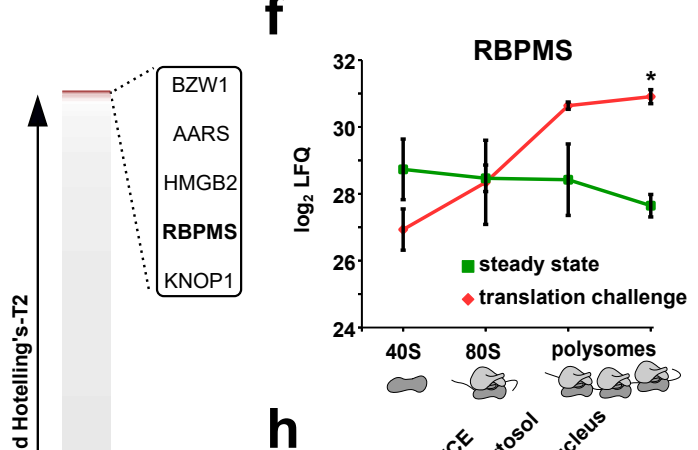

h

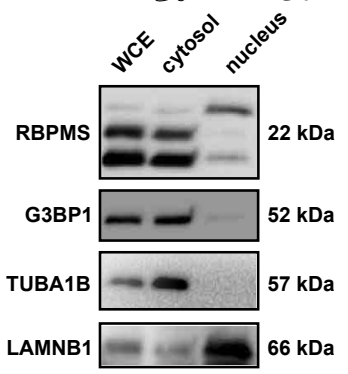

$0^{5} \quad 6^{5} 8^{5}$ polysomes
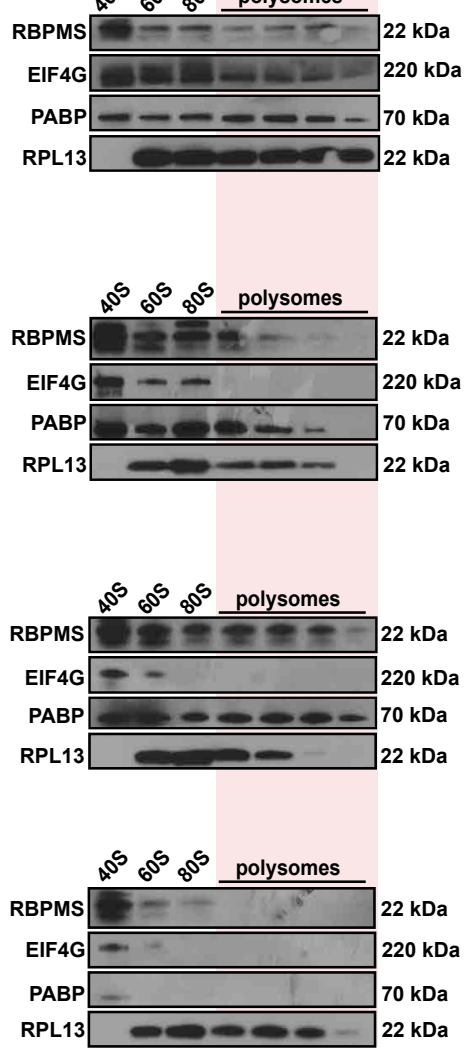

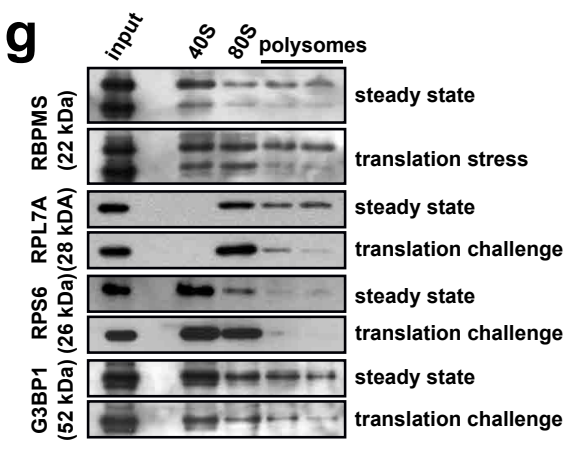


exon1 intron1 bioRxiv preprint doi: https://

Fig. 2
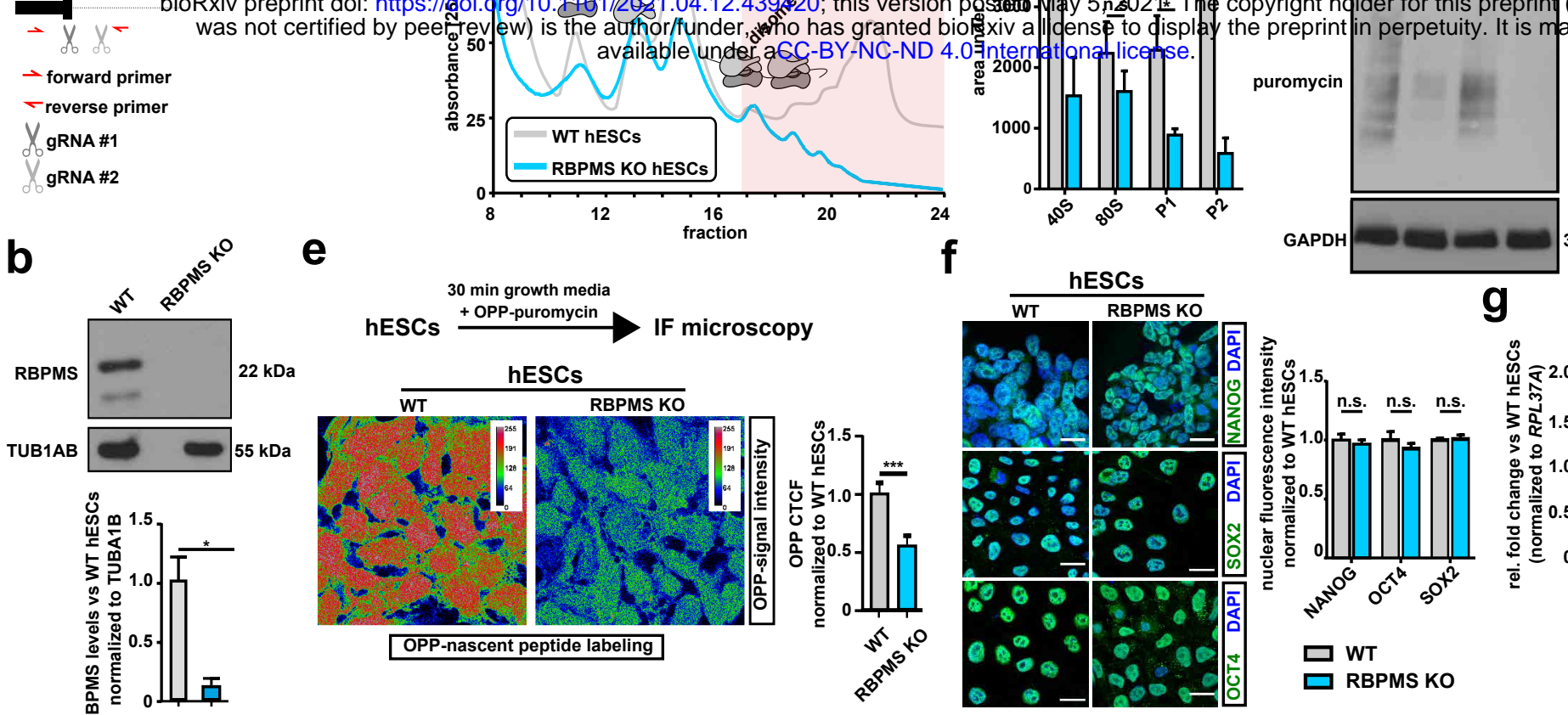

puromycin hESCs $\stackrel{+ \text { OPP-puromycin }}{\longrightarrow}$ IF microscopy
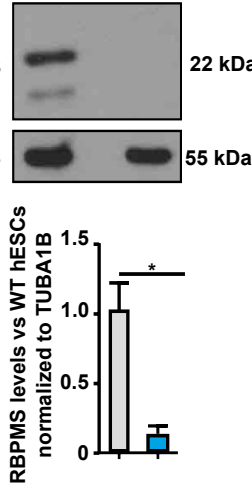

h three lineage differentiation
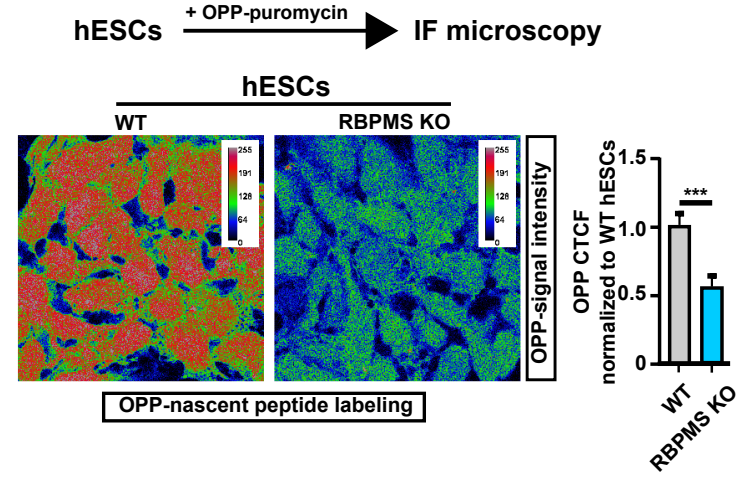

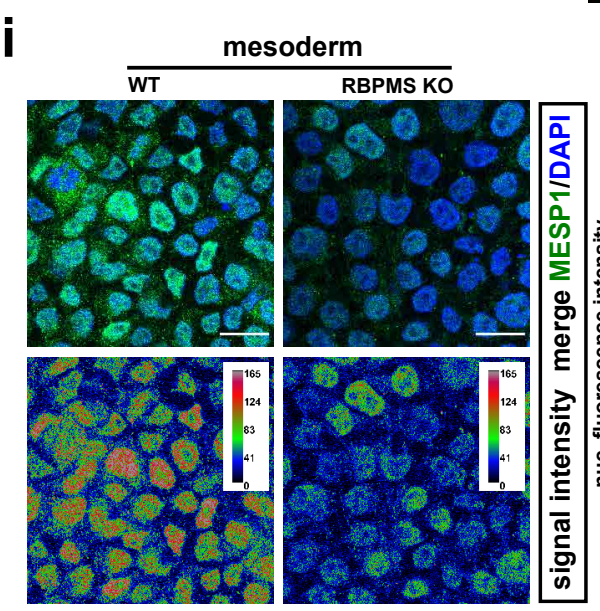

m

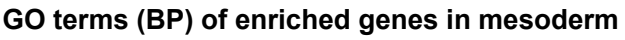

- downregulated in RBPMS KO (mesoderm)

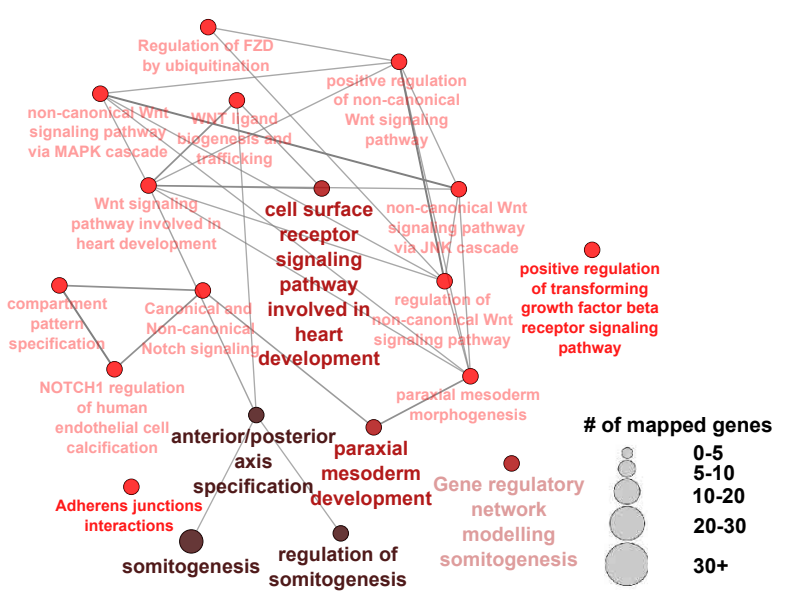

-upregulated in RBPMS KO (mesoderm)

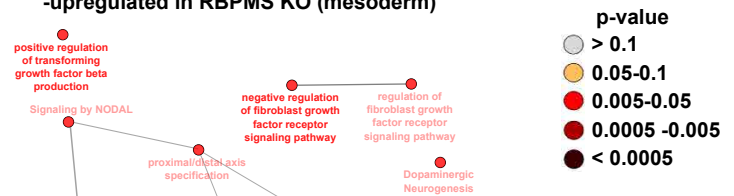

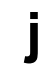

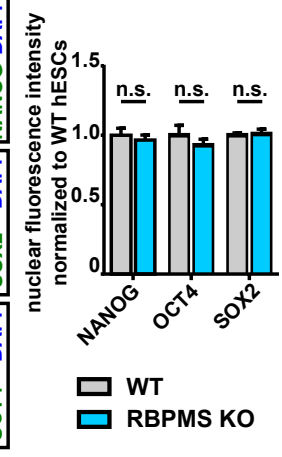

g

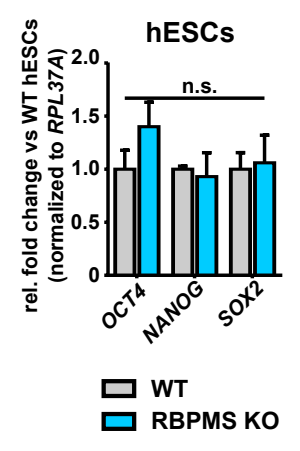

WT vs RBPMS KO hESCs

hESC $\stackrel{\substack{+ \text { BMP4 } \\+ \text { CHIR99021 }}}{\longrightarrow}$ mesoderm

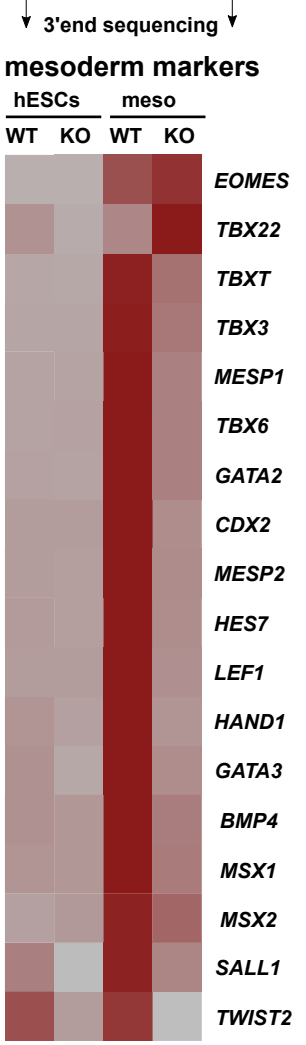

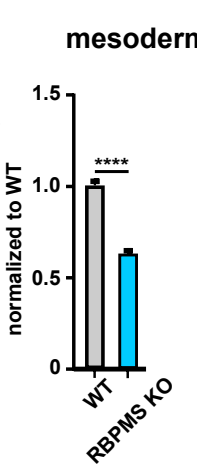

n
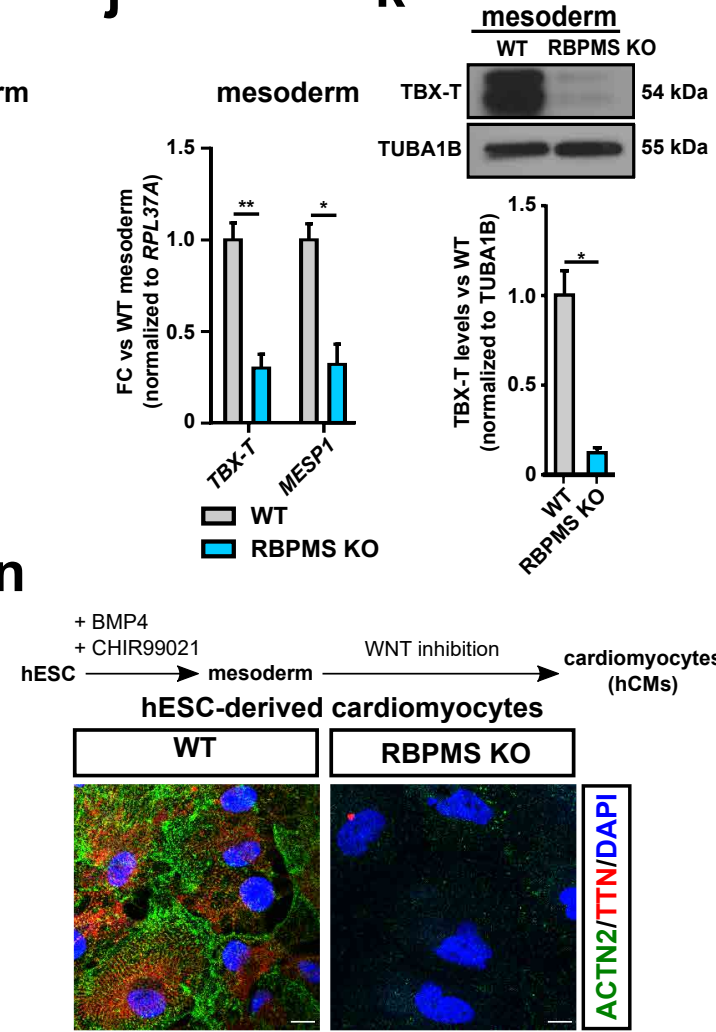

WNT inhibition cardiomyocytes (hCMs)
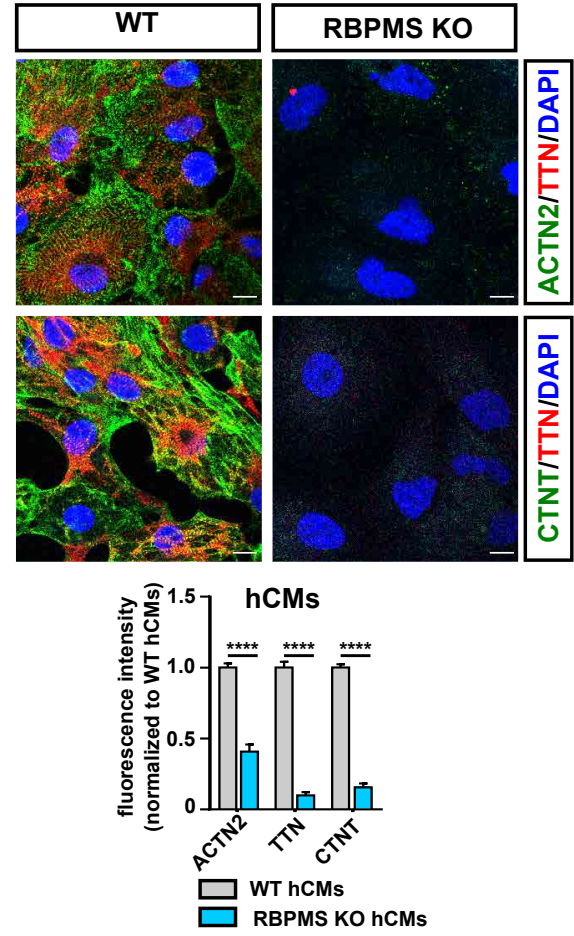
요

G1 $\mathrm{s}$ G2

G1 $\mathrm{S}$

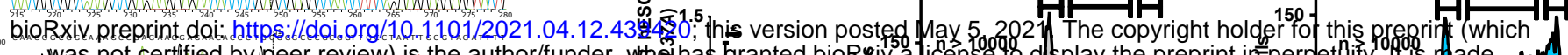
RBPMS Ko hEscs

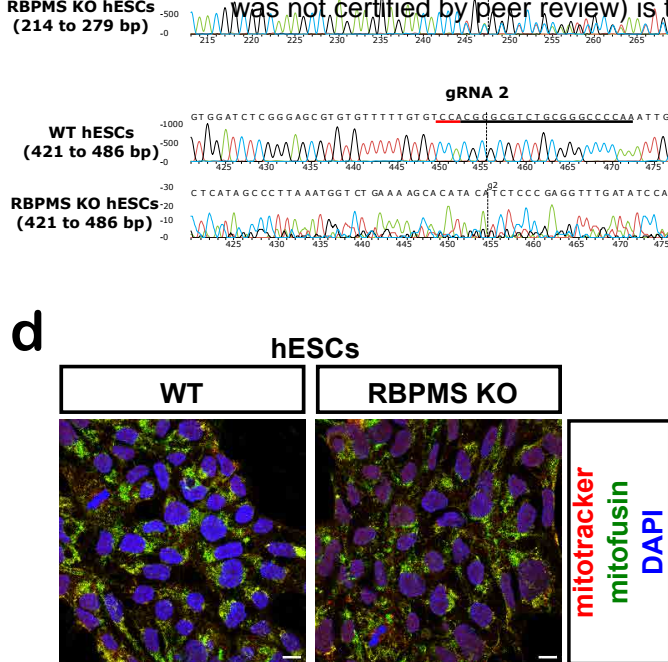

g

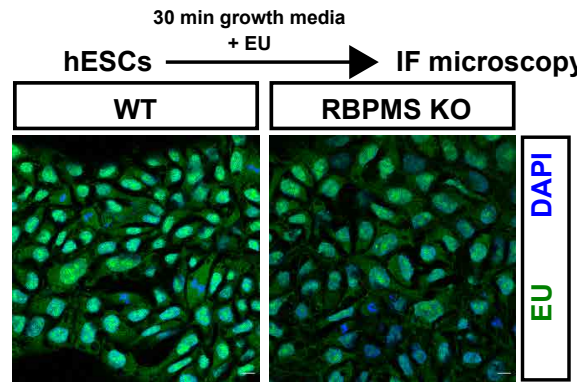

h

30 min growth media

hESCs $\stackrel{+ \text { puromycin }}{\longrightarrow}$ IF microscopy
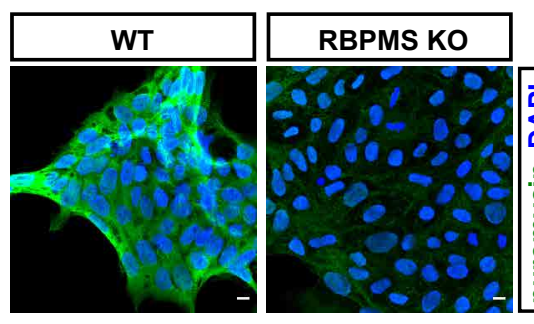

m

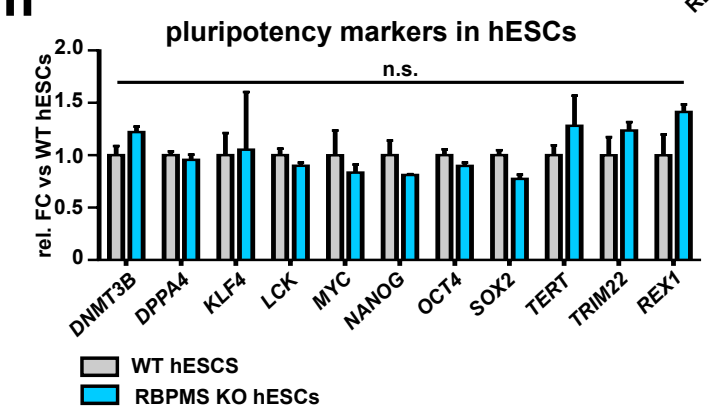

$\mathbf{n}$

ह
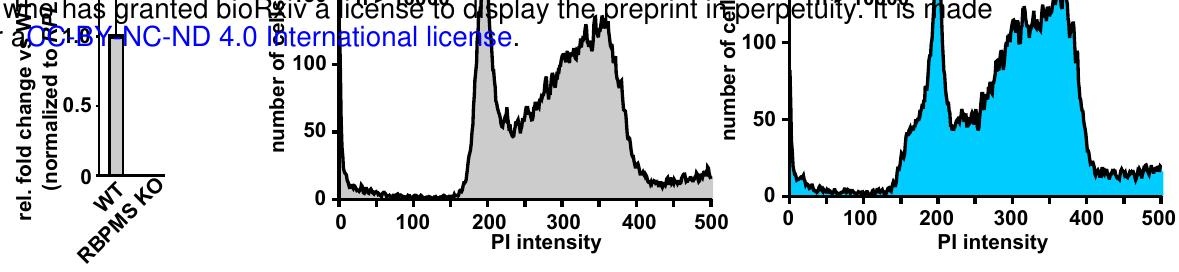

e

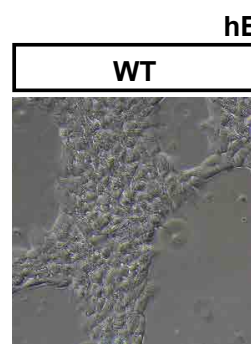

hESCs

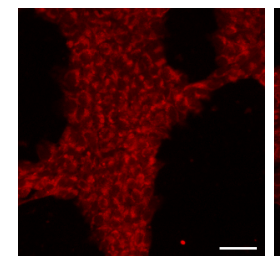

i

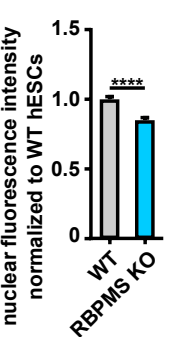

K
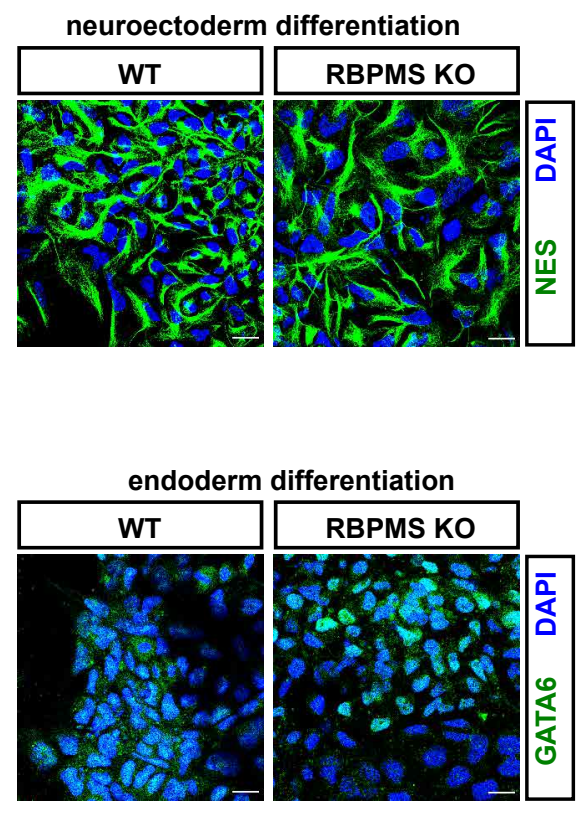

expression of neuroectoderm and endoderm markers after mesdoderm induction neuroectoderm endoderm hESCs mesoderm hESCs mesoderm $\overline{\text { WT KO }} \frac{\text { WT KO }}{\text { WT KO }} \frac{\text { WT KO }}{\text { WT }}$

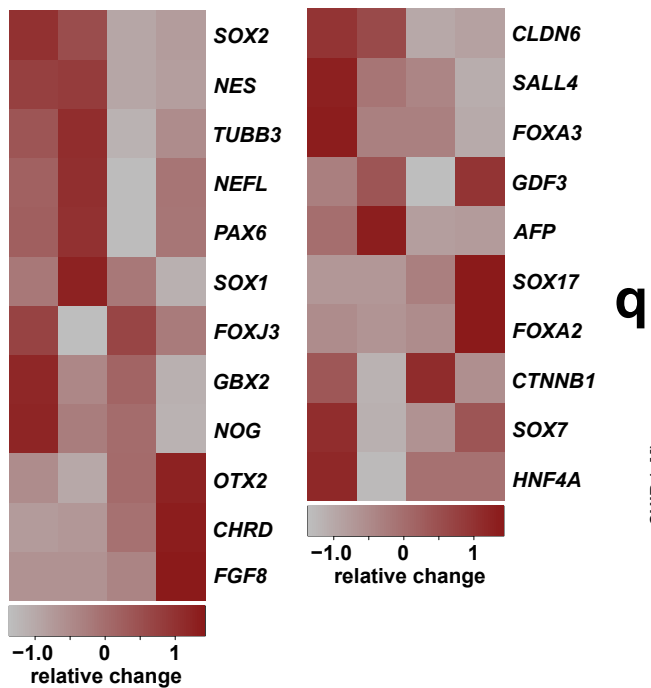

f

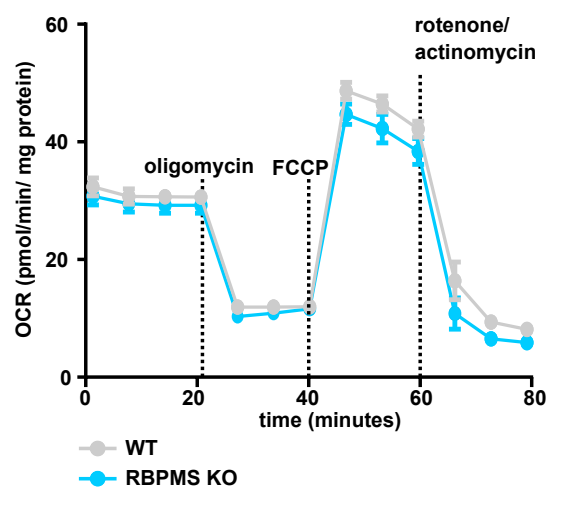

j

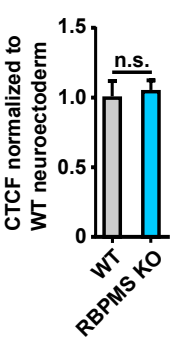

I

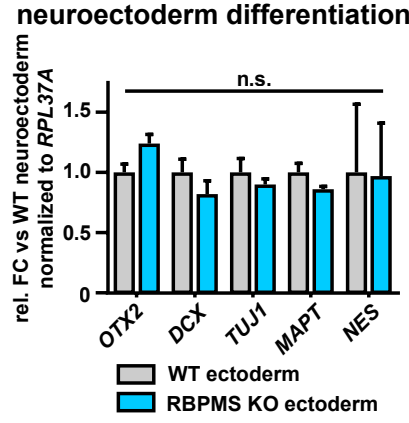

endoderm differentiation

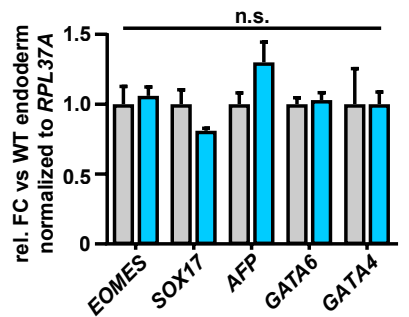

p $\square$ RBPMS KO endoderm

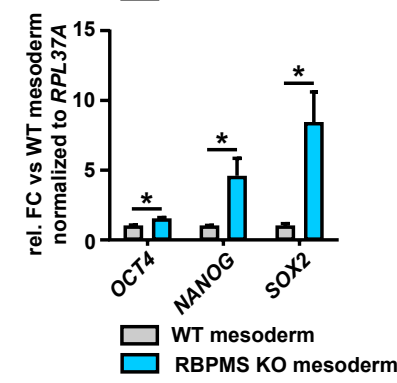

RBPMS KO mesoderm

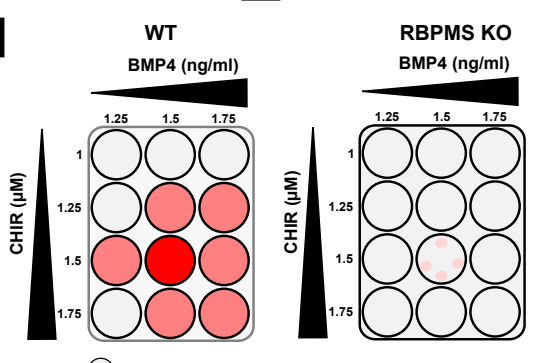

no cardiomyecyes

- $-5 \%$ cardionyocyles

9-50\% cardidomyosyes 
bioRxiv preprint doi: https://doi.org/10.1101/2021.04.12.439420; this version posted May 5, 2021. The copyright holder for this preprint (which was not certified by peer review) is the author/funder, who has granted bioRxiv a license to display the preprint in perpetuity. It is made available under aCC-BY-NC-ND 4.0 International license.

a
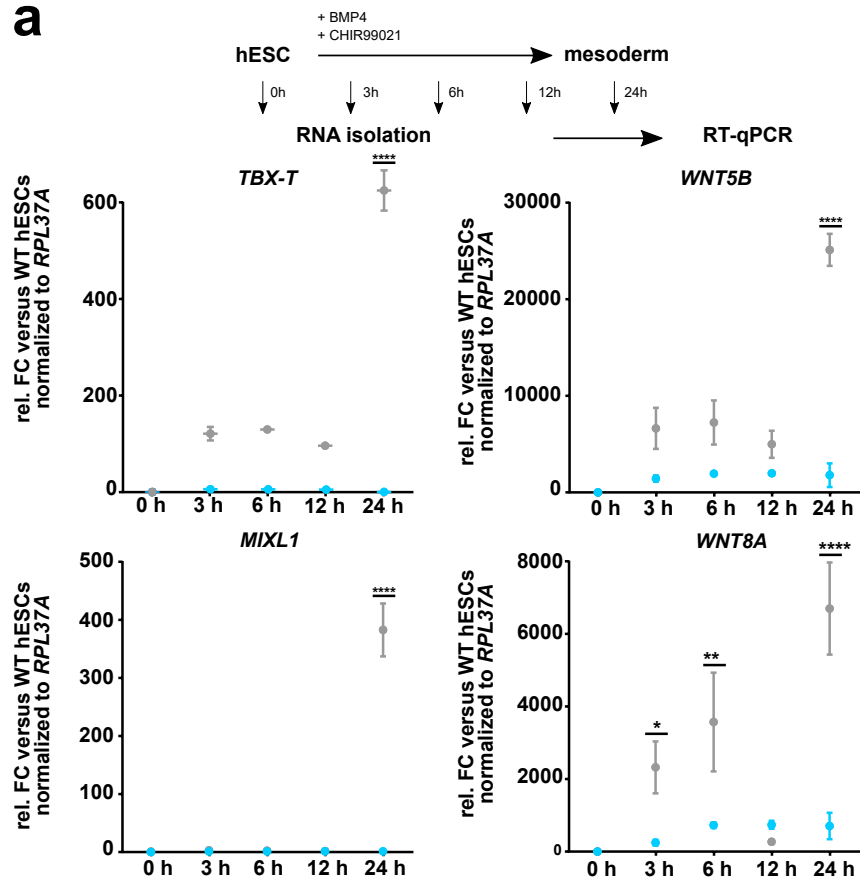

- WT

RBPMS Ko 
b Pearson correlation $\mathbf{C}$ Target enrichment analysis $\mathbf{C}$ Motif enrichment analysis

Fig. 3 (Uv-c $2254 \mathrm{~nm})$

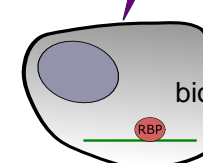

Rxiv preprint doi: us: velojiorg/10.1101/2021 RBP

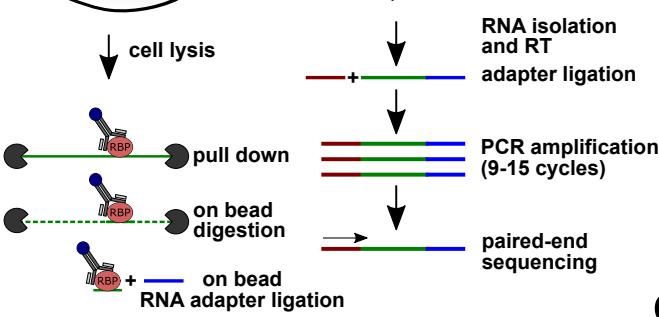

\section{g}
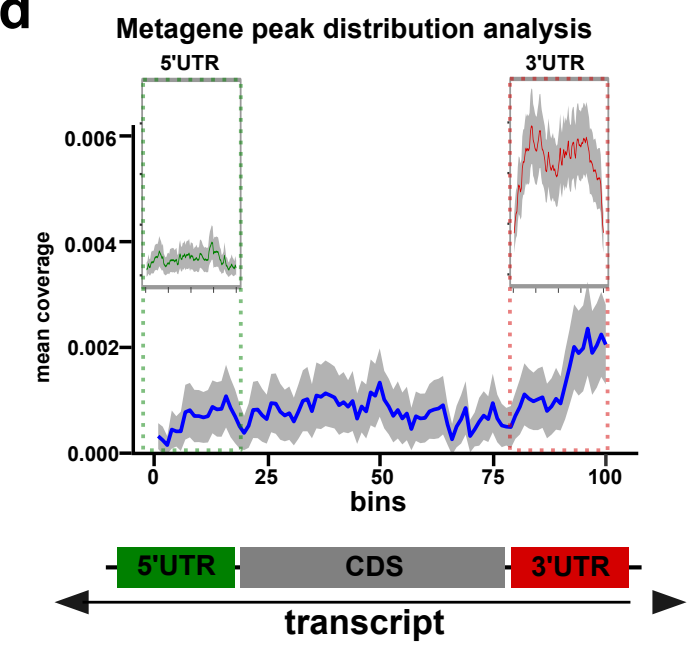

$\mathbf{j}$

two step regression-based clustering of mRNAs in ribosomal fractions repressed in KO

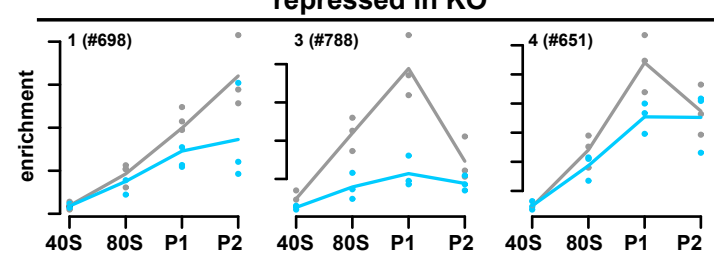

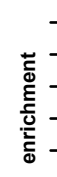
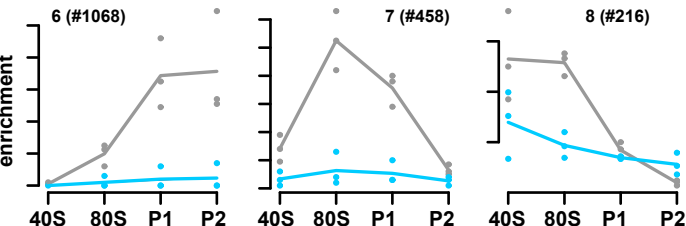

activated in $\mathrm{KO}$
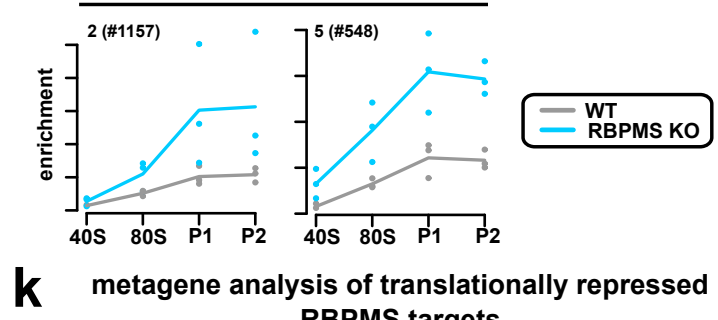

k

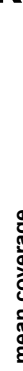

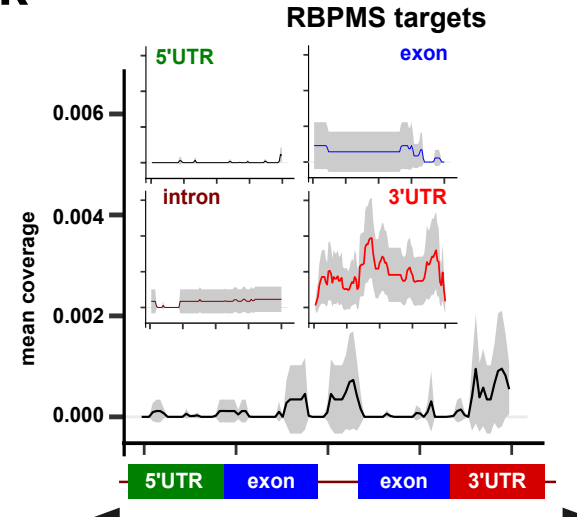

n
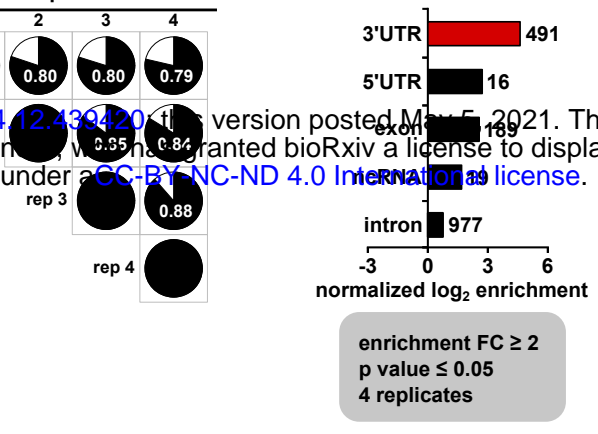

$\mathrm{UCACCUSCAC}$
eCLIP enrichment of curated 3'UTR targets
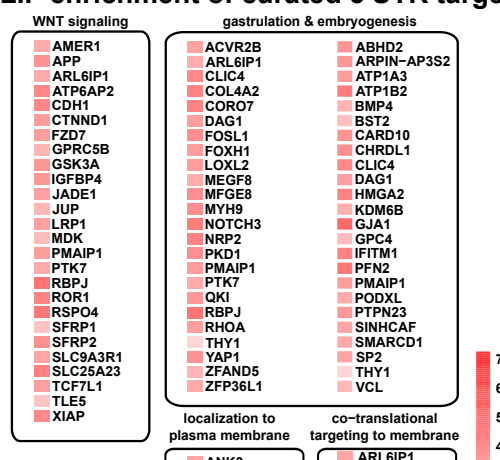

h

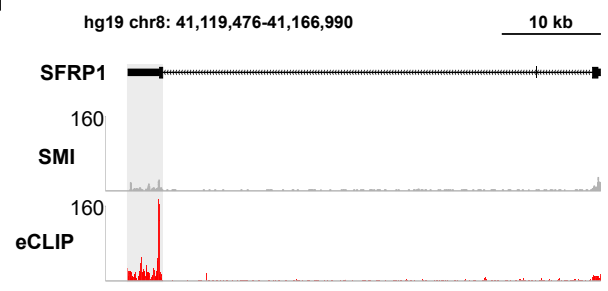

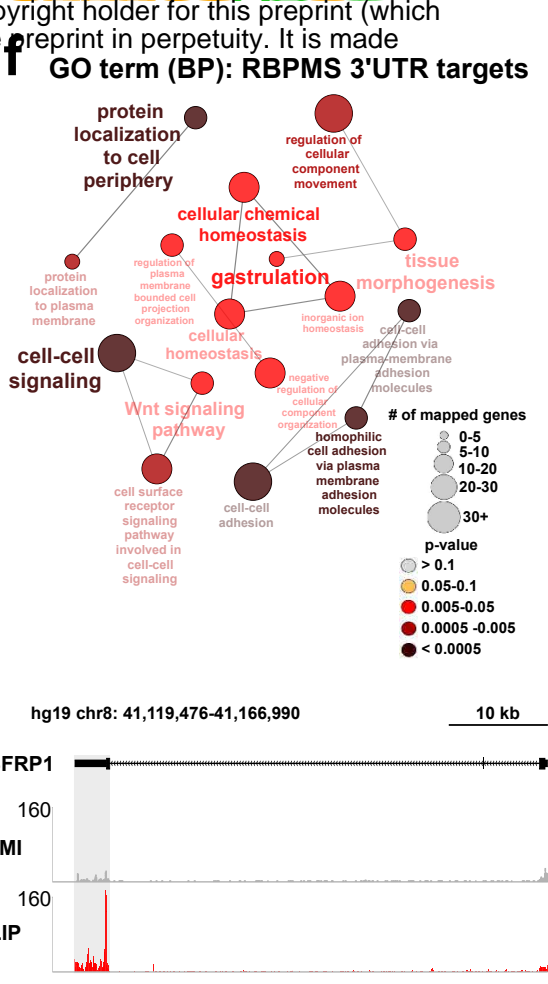

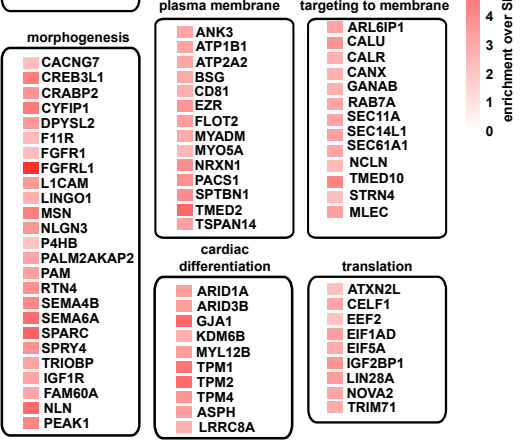

J $\mathrm{GO}$ terms (BP): translationally repressed in RBPMS KO

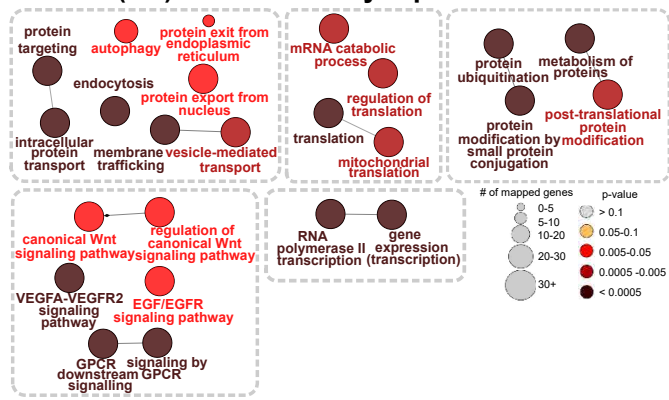

GO terms (BP):translationally repressed 3'UTR targets in RBPMS KO
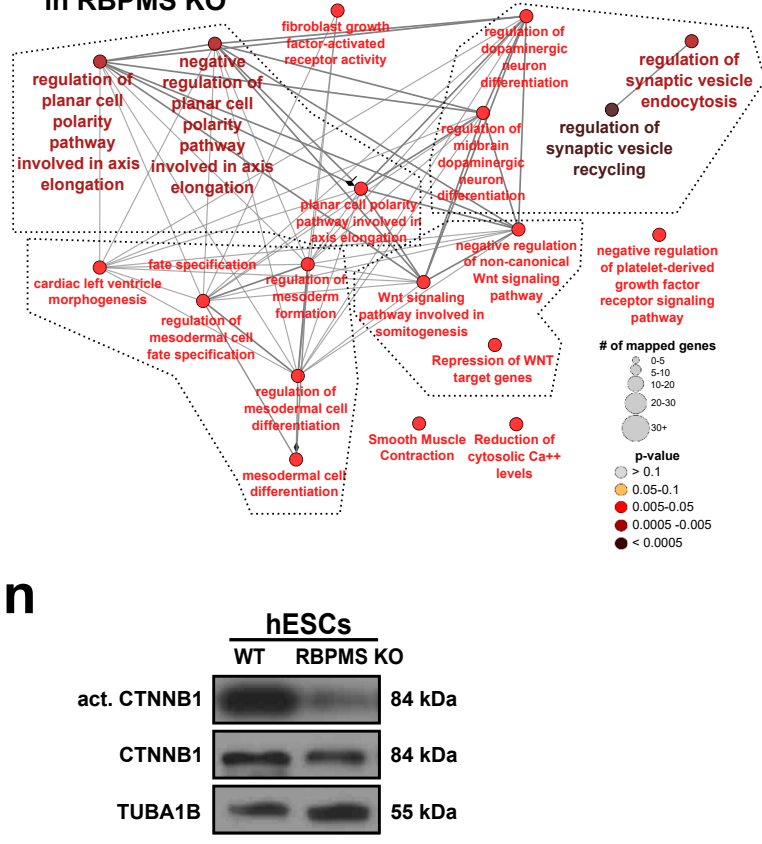

m

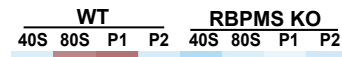

点

종

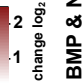

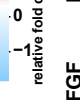



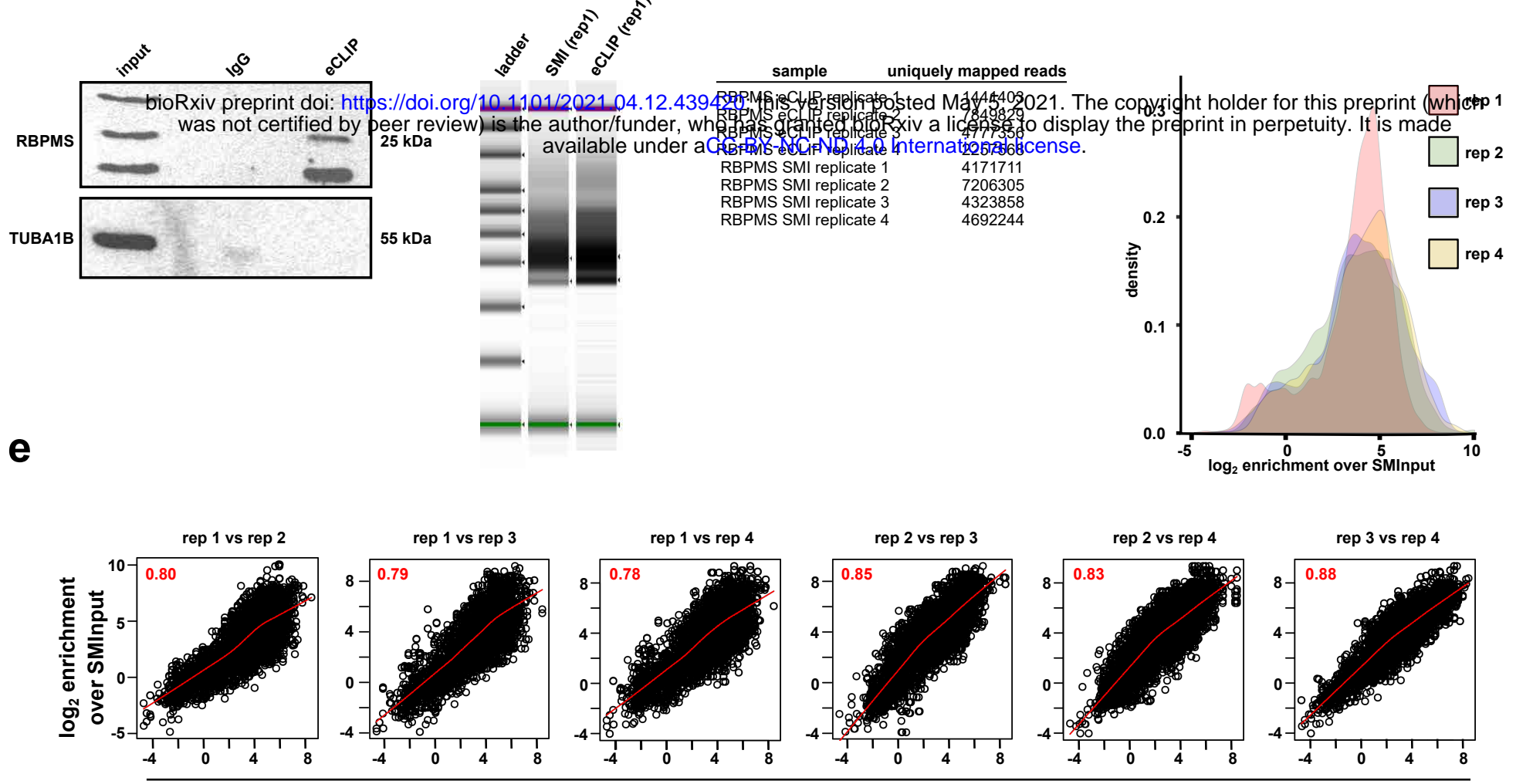

$\log _{2}$ enrichment over SMInput

rep 3

\section{$\triangle C A C$ USCACAC}
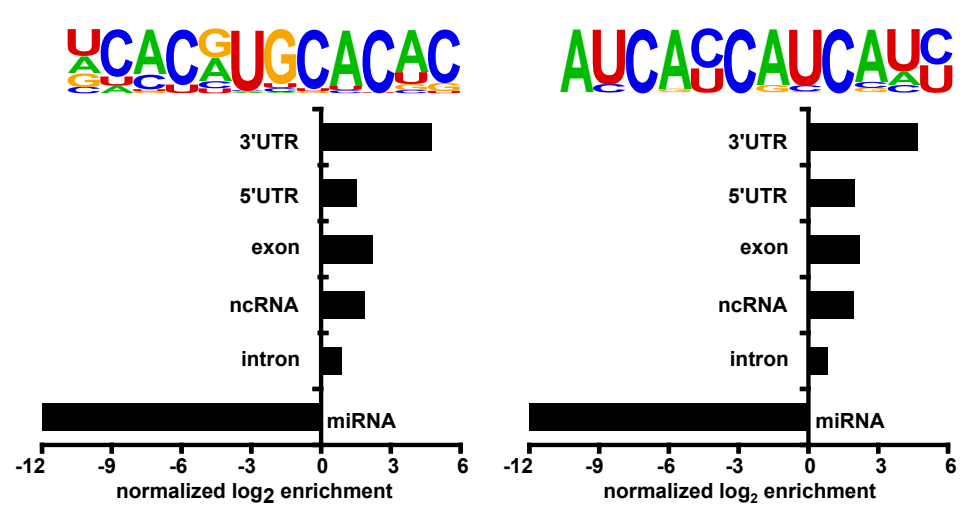

UCACCUSCAC

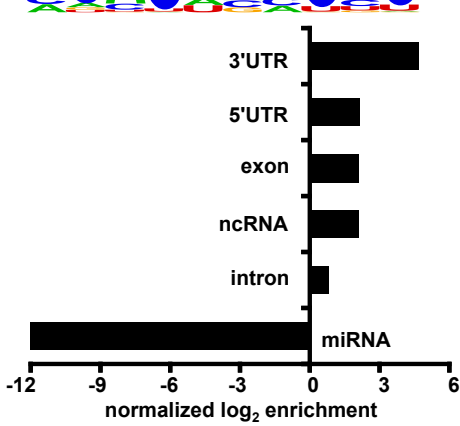

Cellular component 3'UTR targets

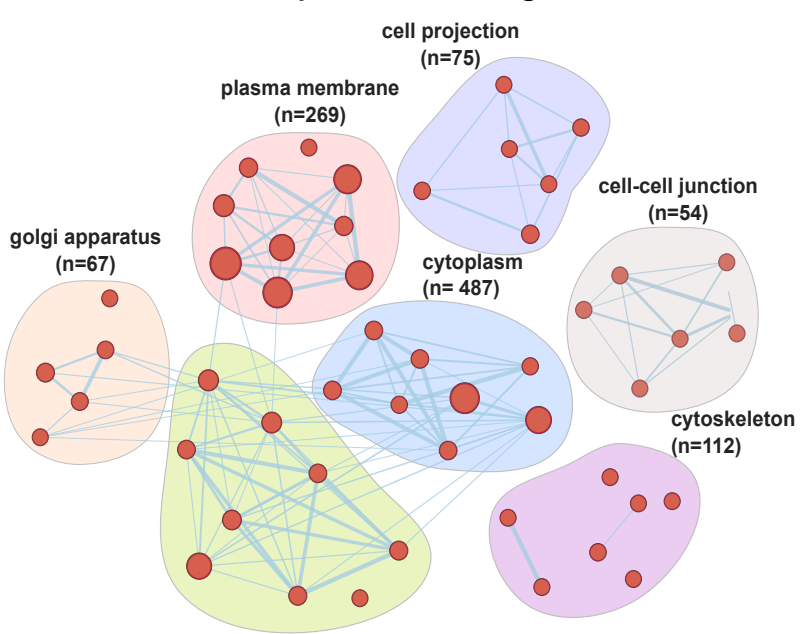

endoplasmatic reticulum ( $n=106)$ 
a translation state RNA-sequencing (TS-seq)

total RNA seq (transcriptome)

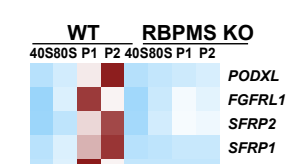

d

Extended Data Fig. 5

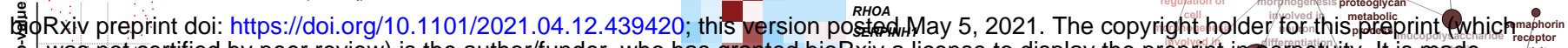
was not certified by peer review) is the author/funder, who has granted bioRexiatra license to display the preprint in perpefuity. It is made

b -transcriptome changes
translatome changes

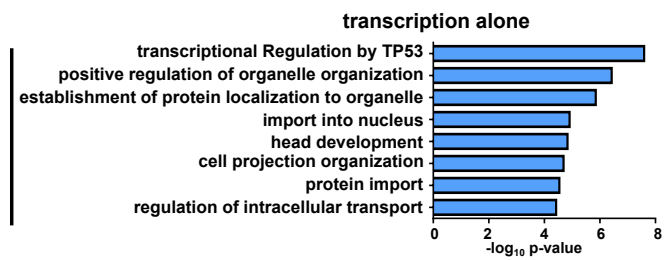

up in RBPMS KO hESCs

e

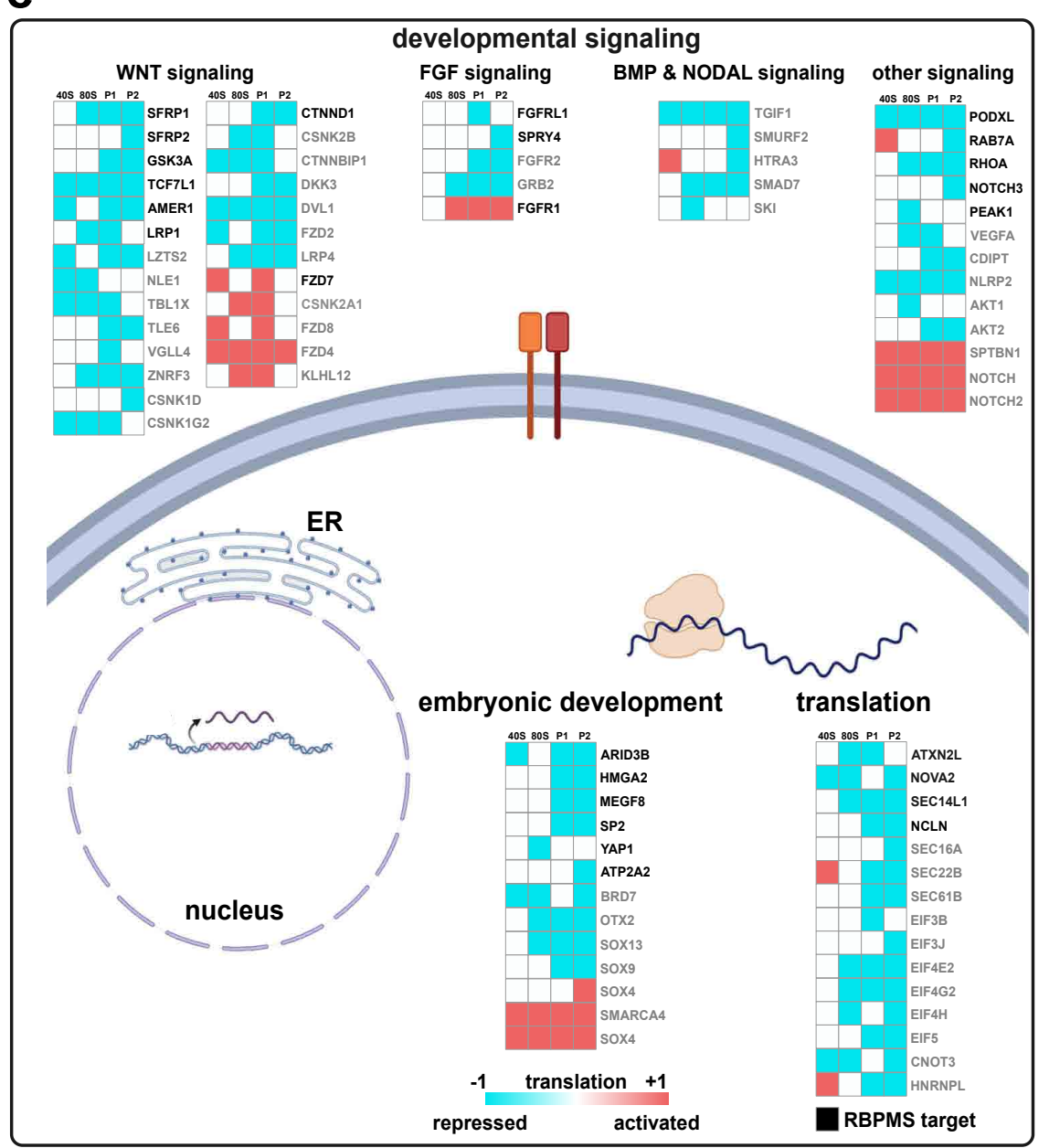

h

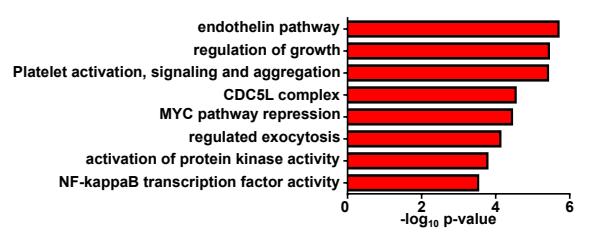
405 80S P1 P2

FFRP2 TCF7L1

MER

ZTS2

NLE1

TLE6

VGLL4
ZNRF3

CSNK1D

CSNK1G

\section{ER}

repressed activated

GO term of depleted 3'UTR targets (70)

(protein abundance quantified by LC-MS)

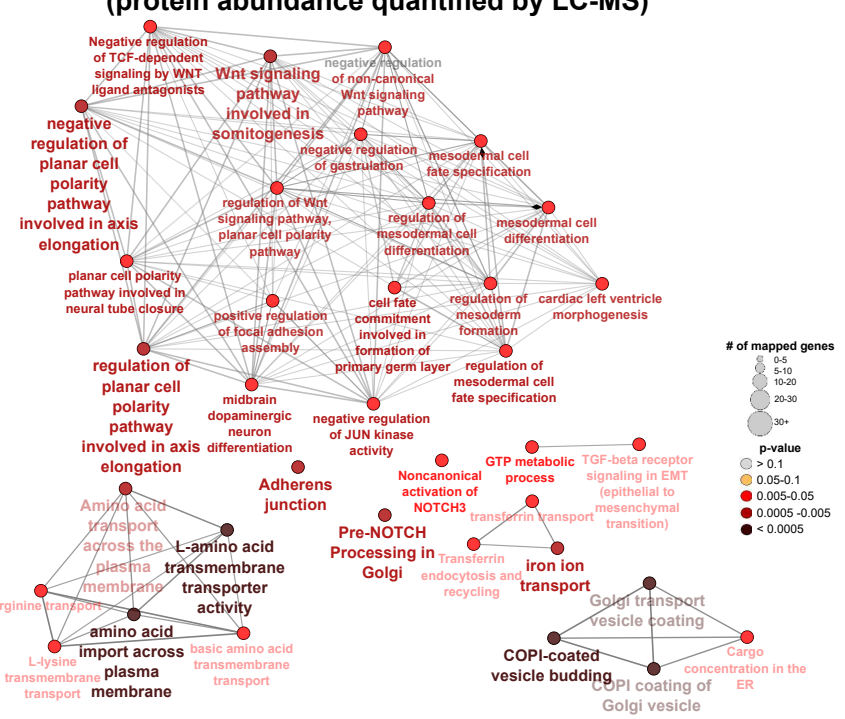

ATP2A2
SLC9A3R1

SLC9A3R1
SLC31A1

SLC25A23

SLC1A5

PKM
P4HB
NOVA2
MEX3A

MEX3A

IGF2BP1

HNRNPC

ATXN2L

ZNF275

ZNF134

TCF7L1

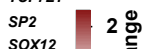

SOX12

NR6A1 1 ¿

\begin{tabular}{l|l} 
HMGA2 & $-1 \frac{2}{\frac{\pi}{0}}$ \\
ARID3B
\end{tabular}

SMARCD1

NACC1

\section{f hESCs}

(WT and RBPMS KO) $\longrightarrow$ whole proteome (LC-MS)

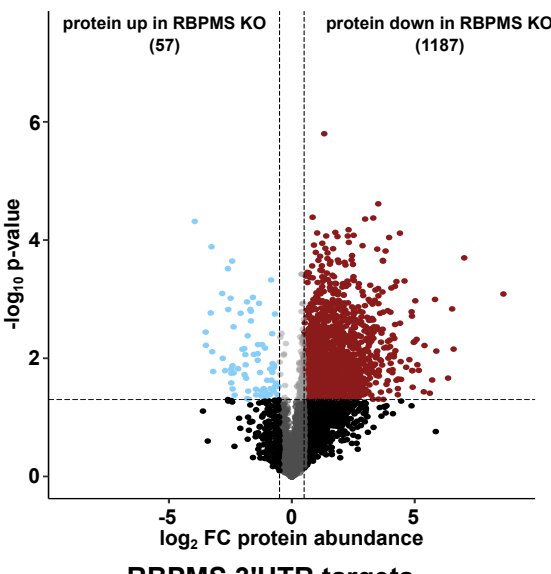

g

RBPMS 3'UTR targets

(protein abundance quantified by LC-MS)

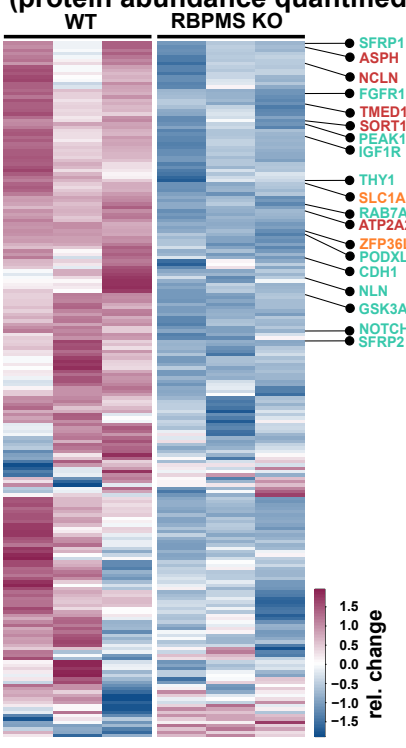

developmental signaling ER/membrane protein synthesis 70/208 depleted $1 / 208$ enriched

mRNA stability in WT and RBPMS KO hESCs
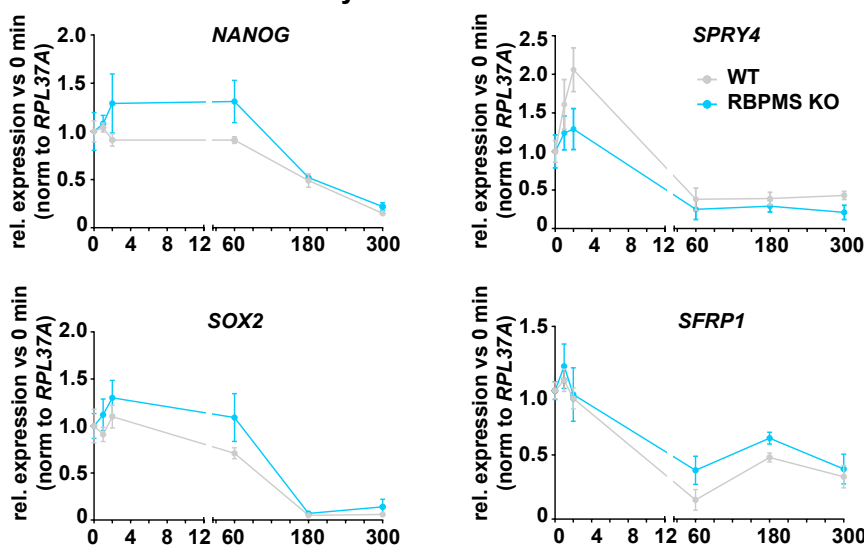
